\title{
Monitoring Mood Disorder Using Activity Data
}

\author{
Rachel Heath \\ School of Psychological Sciences \\ University of Newcastle, Australia
}

\begin{abstract}
A person's mental health depends on how well they cope with environmental demands, from family, work and other potentially stressful situations. We examine the idea that a chronic illness, such as bipolar disorder, can be monitored using a complexity index obtained from a series of behavior measurements, such as activity measured by an ActiGraph. Low health status is associated with a reduction in complexity. In a single case proof-of-concept study, Sample Entropy and Permutation Entropy applied to ten-minute detrended and logtransformed activity data quantified the dynamic changes that occurred over 103 days for a 20-year-old male diagnosed with bipolar disorder. Daily log-log spectra were computed to check for long-term correlation in the data, and day-to-day changes in a logistic transform of the Permutation Entropy estimates were assessed by an adaptive Kalman filter. A significant change in activity was detected approximately three weeks prior to hospitalisation. A change in activity complexity, suggested by changes in the slope of linear log-log spectra, diurnal trends in Sample Entropy and in the transformed Permutation Entropy estimates, occurred in the morning and to some extent in the evening of the 24-hour activity time series. These findings have important implications for preventative mental health, as commercially available activity monitoring devices might function as an automated early warning for relapse in people diagnosed with bipolar disorder.
\end{abstract}




\section{Introduction}

We explore how complexity measures based on nonlinear technologies can be used to monitor a person's wellness over extended periods of time using activity data from people diagnosed with bipolar disorder and their matched controls. The fundamental principle is that good health is characterized by a high level of complexity in behavioral and physiological measures recorded over time, whereas an early sign of a decline in health is indicated by a gradual reduction in complexity. Although the available data are somewhat scant, several single-case proof-of-concept studies have indicated that serious relapse in mental health is accompanied by a decrease in complexity of time series acquired using wearable devices. This decline in health is indicated by a reduction in entropy as measured by techniques such as Sample Entropy and Permutation Entropy.

We begin with some background information on complexity as it relates to mental health. Then we analyse activity data for people diagnosed with Bipolar disorder. These analyses show how it might be possible to use wearable devices to detect the early signs of a mental illness relapse to allow timely intervention that might avoid adverse consequences, such as admission to hospital with its associated costs to the person, their family and the community.

\section{Complexity and Mental Health}

The complexity of mental health is revealed by the complicated network structures that bind the various symptoms people report for the various mental disorders. Collectives of these symptoms can be linked in unexpected ways to offer a new insight into the diagnoses of mental illness. For example, major depression can be characterised by extra morning sleepiness and an increase in weight. These changes can often be accompanied by dysregulation of other bodily systems, especially those associated with the inflammatory response (Boschloo, van Borkalo, Rhemtulla, Keyes, Borsboom \& Schoevers, 2015). 
The study by Boschloo et al. used data from a large community sample (National Epidemiologic Survey on Alcohol and Related Conditions, NESARC, N = 34,653) involving the 120 psychiatric symptoms contained in twelve major DSM-IV diagnoses. The resulting network is shown in their Figure 1. Each subnetwork representing a diagnosis is linked to several other networks involving other diagnoses. Of interest, a major depressive episode has only $68.4 \%$ of the symptoms related to its DSM-IV diagnosis, but it has many links to other diagnoses in the network, such as Dysthymia, Generalized Anxiety Disorder, Mania and Post-traumatic Stress Disorder.

Borsboom (2017) proposed a general network model for the dynamics of mental illness resulting from the collective interactions between symptoms and their biological and environmental influences. If the interactions are strong enough, the dynamics becomes selfsustaining leading to mental illness. This undesirable situation results perhaps from a disconnect between the person and their environment, so that the complexities of both are incompatible. Any stable state emerging from this person-environment interaction leads to a reduction in the complexity of the corresponding isolated activation network. The consequence may be the onset of psychopathology. Any relief for the person requires the original complexity of the person-environmental interaction to be restored.

A within-person symptom network contains symptoms at each node. The links between nodes represent interactions between the symptoms leading to combinations that conventionally define a mental illness diagnosis. Importantly, the systems can be connected to environmental events, including physiological processes, such as the autonomic nervous system, that play an important role in mental disorder genesis. These environmental events form their own complex network of influences. 
According to this conceptualisation of mental illness, one's mental health is determined by the pattern of activation that flows through the symptom network. If persistent clusters evolve, these may be associated with a stable state of mental ill-health. Also, the strong interactions between symptoms within a cluster and with possibly weaker symptoms outside the cluster, ensure that there are comorbid conditions that accompany mental illnesses. For example, intermediate symptoms such as insomnia, fatigue and poor concentration can link post-traumatic stress disorders and major depression. It is the selfsustaining property of the symptom network dynamics that ensures that the illness persists long after the environmental triggers have terminated, as is common in these latter conditions. This is an example of hysteresis, persistence of the current state of a system without immediate return to previous states. The current state plays an important role in the maintenance of a mental illness, provided the network contains strong connections that reduce its overall ability to adapt appropriately to potentially favourable environmental influences.

In terms of this network representation, mental health occurs when the network connections are weak and stable, a state to which the network returns when it has been released from environmental stressors. This is characteristic of resilient people who can readily deal with adverse events. Vulnerable individuals, on the other hand, are at risk of returning to a strongly connected symptom state. These characteristics define mental illness risk. So weak environmental stressors and a weak network connectivity are conducive to good mental health, whereas overly strong associations between these variables can lead to mental illness, a maladaptive person-environment contingency.

Our first case study to illustrate the application of wearable technology to the monitoring of mental illness uses an ActiWatch to monitor a patient's movements 24/7. This 
is an example of a technique known as actigraphy that is commonly employed in hospital sleep studies. The use of actigraphy for predicting dynamic fluctuations in potentially psychopathological behaviour may be more generally applicable than appears at first sight. An interesting small-world network analysis of the contagion of all diagnoses contained in DSM-IV (Borsboom, Cramer, Schmittmann, \& Waldorp, 2011), a precursor of the study reported by Boschloo et al. (2015), produced just four diagnostic "hubs" (by analogy with airline route networks), insomnia, with 71 connections to other symptoms, psychomotor agitation (68 connections), psychomotor retardation (61 connections) and depressive mood (60 connections). These four diagnostic "hubs" subsume 208 other symptoms in DSM-IV as well as 69 separately described disorders, a massive compression of diagnostic information (see Table 1 in $\mathrm{Hu}$, Scheer, Ivanov, Buijs \& Shea, 2007). It is of considerable interest that all four diagnostic "hubs" can be assessed using actigraphy. Hence the proposed technology presented in this paper may have wide application in the monitoring of substantial aspects of psychopathology. A similarly detailed network study of DSM-V symptoms has not been completed.

\section{Physical Activity and Mental Health}

In a study conducted in 1992-1993, regular exercise was reported by about $60 \%$ of a large sample of American adults aged 15 to 54. Although regular exercise was associated with a reduced likelihood of major depression and phobia, there was no significant relation to generalized anxiety (unless comorbid) and bipolar disorder (Goodwin, 2003). Janney, Fagiolini, Swartz, Jakicic, Holleman and Richardson (2014) had 60 adults diagnosed with bipolar disorder wear an ActiGraph on the hip for seven days and compared their activity with that of 60 matched controls. The ActiGraph is a device that measures a person's activity, similarly to the ActiWatch that we used in our example application. For these people, most of whom were in a euthymic or well state during the observation period, activity was mostly 
sedentary $(78 \%)$, this being significantly greater for the bipolar patients than controls. So, measuring activity using wearable devices may provide useful information on the progress of chronic disorders, such as depression.

\section{Activity in People at Risk of Bipolar Disorder}

Bullock and Murray (2014) investigated changes in activity and circadian rhythm in people who were either at high or low risk for bipolar disorder. The tendency for people to gravitate towards high or low levels of mood is indicative of a two-dimensional view of depression and mania in people diagnosed with bipolar disorder. This view of mood can be assessed in general populations using the General Behavior Inventory $(G B I)$ that provides separate measures of mania and depression. The $G B I$ is one of the few instruments that are worth considering for prognostic evaluations of bipolar risk, the total $G B I$ score providing quantitative evidence of a person's vulnerability to bipolar disorder (Ratheesh, Berk, Davey, McGorry \& Cotton, 2015).

In Bullock and Murray's study, those in the top and bottom $10 \%$ of scores on the $G B I$ were selected for an actigraphy study lasting seven days with 36 people in each group, 75\% of whom were female with a mean age of 22 years. People at risk of bipolar disorder are likely to have unstable circadian rhythms, and their variable lifestyle can lead to variations in their social rhythms. As attitudes towards achievement, dependence and self-control are predictive of relapse, these people also completed the 24-item Dysfunctional Attitudes Scale.

Relative activity during any 24-hour period was measured as the difference between activity in the most active 10-hour period and in the least active 5-hour period divided by the total activity in that 24-hour period. An important finding from the study was a significantly lower relative activity for high-risk people with a previous history of a depressive episode, even though there was no difference in overall activity between the two groups. The elevated relative activity in the high-risk group was reflected mostly in greater activity in the evening. 
However, it is worth noting that a large national study in the USA showed no relationship between bipolar disorder and any variation from the norm in overall physical activity (Cairney, Veldhuizen, Faulkner, Schaffer \& Rodriguez, 2009). We will reanalyse these activity data using complexity methods.

Psychomotor retardation, a dramatic reduction in movement, is common in about $40 \%$ of young people with severe depression. Finazzi, Mesquita, Lopes, Fu, Oliveira and Del Porto (2010) used actigraphy to measure activity in six adolescents, mean age 16 years, who had been diagnosed with depression and offered antidepressant medication. Corroborative information about progress was obtained by weekly scores on the Children's Depression Rating Scale Revised (CDRS-R). Measures of activity over nine weeks were obtained using an ActiWatch. There was a significant negative correlation between the mean activity and depression score over the nine weeks, indicating that the measured reduction in depression was associated with an increase in mean activity level. This finding was only statistically significant for activity recorded between noon and $6 \mathrm{pm}$.

Although medication is the most common regular treatment for bipolar disorder, a person's progression from one stable state of the disorder to another is not always monitored, except for subjective reports using a mood diary. These data are not often used to indicate that medical intervention may be needed due to the imminent onset of an adverse phase of the disorder, such a major depressive phase or a manic relapse. The use of passive monitoring using wearable data is a promising way to monitor a person's progress so that adverse situations are minimised, especially the maladaptive behaviour that often leads to long periods of hospitalisation.

\section{Activity Measurement}

In their review of activity and sleep assessment, Ancoli-Israel, Cole, Alessi, Chambers, Moorcroft and Pollak (2003) suggested that actigraphy provides a more accurate 
quantification of activity during sleep periods than during awake periods when compared with sleep assessment performed using polysomnography (PSG), the commonly used gold standard in sleep studies. PSG involves measurements of brain activity, eye movements, muscle activity and heart rhythm. Despite its comparative simplicity, actigraphy provides measurements of total sleep time that correlate over 0.9 with PSG, measurements being more reliable for healthy adults rather than those with sleep disturbances that were already diagnosed using PSG. Estimates of sleep and awake times tend to become less accurate as sleep efficiency decreases.

Actigraphy can also be used to estimate circadian rhythm. Traditional measurement involves fitting a cosine curve to the activity data yielding measures of amplitude and period, one simple measure being the difference in activity between the ten most active hours and the five least active hours during the day. This is expressed as a ratio of the total activity for that day. This measure has also been shown to be diagnostic of bipolar disorder risk (Bullock \& Murray, 2014).

Minassian, Henry, Young, Masten, Geyer and Perry (2011) measured the exploratory behaviour of hospitalised patients diagnosed with a manic episode and compared their activity with that of healthy controls. Exploratory and novelty-seeking behaviour was assessed in a human version of the animal open-field test, the Behavioral Pattern Monitor. The bipolar patients exhibited greater exploratory behaviour than controls, a finding that was corroborated by a greater level of dynamic entropy, or complexity, of their psychomotor movements. For the bipolar subjects, there was an habituation effect with gradually less exploratory behaviour being observed over three successive sessions. This effect was probably due to an increase in antipsychotic medication during treatment. The controls exhibited exploratory behaviour that remained significantly lower and relatively more stable 
than that of the bipolar patients. These results suggest that activity measurement can be useful for monitoring the progress of people diagnosed with a major mental illness.

\section{Sleep Abnormalities in Bipolar Disorder}

Geoffroy, Scott, Boudebesse, Lajnef, Henry, Leboyer, et al. (2015) conducted a meta-analysis of studies measuring the sleep quality experienced by bipolar patients in remission. The importance of monitoring sleep when the patients are well is evident when sleep disruption acts as a possible precursor for the next episode that may require hospitalisation. The four measures investigated were sleep latency, sleep efficiency, sleep duration and wake-aftersleep-onset (WASO), defined by the amount of time awake between sleep onset and eventual awakening. The meta-analysis reviewed nine eligible studies containing 202 bipolar cases in remission and 210 controls. The median duration of studies was quite short at seven days. Compared with the controls, bipolar disorder was associated with significantly longer sleep latency, longer sleep duration, longer WASO and less sleep efficiency.

Using 26 currently well people who had been diagnosed with bipolar disorder and 29 gender and age matched controls, Geoffroy, Etain, Sportiche and Bellivier (2014) used actigraphy recorded over 21 days with an ActiGraph to show that when compared with controls, people diagnosed with bipolar disorder were more likely to report poorer sleep quality and efficiency, more frequent sleep disturbances and more daytime dysfunction. It was noteworthy that the bipolar participants were more prone to depression than the controls, whereas there was no difference in mania symptoms observed during this relatively euthymic phase of the disorder. Actigraphy indicated greater sleep duration and latency, less sleep efficiency and slightly less inter-day stability for the bipolar patients. When measured over days, time in bed, sleep duration, sleep efficiency and fragmentation index, an indicator of the amount of sleep disruption during the night, were more variable for the bipolar patients. The most discriminating variables leading to a classification accuracy of $89 \%$ for bipolar patients 
when compared with controls were sleep duration, sleep latency, sleep fragmentation index variability, a measure based on the proportion of awakenings during stage 1 sleep compared to the total sleep time, and rated daytime dysfunction, this being one of the most discriminating variables. The authors suggested that daytime dysfunction may not be related to sleep alone, but might represent the overall difficulties bipolar patients face in their daily lives.

Kaplan, Talbot, Gruber and Harvey (2012) compared sleep characteristics in 27 bipolar patients and their matched controls using actigraphy (Actiwatch), PSG and sleep diaries. Previous studies have shown that over $50 \%$ of bipolar patients experience some form of sleep disturbance even during their euthymic phases, severe sleep disruption being a common prodrome for a manic episode. There was general concordance between measures of sleep onset latency, WASO, number of awakenings, total sleep time and sleep efficiency between PSG and actigraphy recordings (sleep-wake threshold $=40$ activity counts per minute). This agreement among sleep measures was much greater than that between PSG and subjective reports recorded in a sleep diary. PSG and actigraphy were closest in their respective measures of total sleep time $(r=0.92)$ but had a much lower relationship with sleep onset latency $(r=0.37)$. Of interest, there was no difference between bipolar patients and controls in any of these sleep measures recorded over a 48-hour period.

It has long been recognised that the mathematics of complex systems may provide a tool for describing and predicting, and therefore better managing, the erratic and potentially catastrophic behavioural variations that characterise bipolar disorder (Gottschalk, Bauer \& Whybrow, 1995). Nonlinear approaches of various kinds have been applied to either selfreported mood (Moore, Little, McSharry, Goodwin \& Geddes, 2014; Moore, Little, McSharry, Geddes \& Goodwin, 2012), or objectively measured activity (Indic, Salvatore, Maggini, Ghidini, Ferraro, Baldessarini \& Murray, 2011). Advances in technology indicate 
how useful it might be to apply these techniques to help people. These days, many people throughout the world carry devices that can readily capture, process and interpret their realtime functioning with a resolution capable of allowing complex medical decisions. In an example presented in this paper, one such mathematical approach is presented, based on a unique dataset containing a person's activity in the weeks prior to a manic relapse.

As we have seen, bipolar disorder is a severe psychiatric condition with a lifetime prevalence of about 2\% (Merikangas, Jin, He, Kessler, Lee, Sampson, et al., 2011). The disorder is characterised by episodes of severe depression and mania interrupted by euthymic or well periods of varying duration. Bipolar disorder generates significant morbidity and mortality for the individual, disruption for friends and family, and a significant financial burden due to loss of employment and caring responsibilities. Bipolar disorder, like many other mental illnesses, also incurs a huge cost to health care systems. People diagnosed with bipolar disorder are more likely than those with unipolar depression to develop psychotic and other severely debilitating symptoms that require frequent hospitalisations (Goodwin \& Jamison, 2007).

Although the burden from bipolar depression can be extremely debilitating, manic episodes are the most functionally impairing phase of the disorder, frequently accompanied by psychosis, hyperactivity and sleep disturbance that frequently require expensive hospitalisation (Grunze, 2010). If a mood episode is identified early, timely intervention for mania may obviate the need for hospitalisation. Minimal behaviour changes, such as reducing the amount of environmental stimulation and the use of suitable medication to improve sleep may reduce the chances of a manic episode. Perhaps the use of technology can allow people to identify their own early signs of an episode and obtain timely medical assistance. When this is possible, those suffering a manic episode may experience better outcomes (Lam \& Wong, 2005). 
Current treatment guidelines for bipolar disorder encourage daily mood monitoring to support early intervention. However, this strategy is not ideal because of its demands on people and its unreliability due to a person's possible impaired insight. Research has suggested that 24-hour activity monitoring using a wearable device has the potential to provide automated, objective information about episode precursors (Bullock, Judd \& Murray, 2014).

A characteristic feature of mood disorder is the observed fluctuations in activity levels exhibited by patients as their mood changes. One of the early signs of depression, for example, is a reduction in activity level as the patient withdraws from their usual activities and leads a more sedentary life. On the other hand, incipient mania typically appears as increased physical activity and decreased sleep (Murray, 2010). Indeed, changes in goaldirected activity are now recognised alongside mood changes as a core diagnostic feature for mania (American Psychiatric Association, 2013). In the present theoretical context, this might imply that a reduction in the complexity of activity measures obtained from wearable devices might serve as a precursor for the start of a mental illness relapse

In the next section, we discuss how information obtained from modern wearable devices can be used to monitor a person's wellness over extended time periods. In these applications we use activity measures to inform a model representing the complex processes that subsume people's transitions from well states to possibly unwell states. Statistical decision-making methodologies can then be used in a similar way to industrial quality control to detect the significance of these changes so that the user and nominated others can be advised appropriately. 


\section{Application of Sample Entropy and Permutation Entropy Models to}

\section{Activity Data}

Analyses illustrating the application of Sample Entropy and Permutation Entropy were conducted using ActiGraph activity data provided by Professor Greg Murray from the Brain and Psychological Sciences Research Institute, Swinburne University of Technology, Australia.

Many people carry devices to support the analysis of complex features of their activity that can be used for early detection of relapse, such as the start of an episode in bipolar disorder. One such device, a wrist-worn ActiGraph, was used to track activity from a person diagnosed with bipolar I disorder who was eventually hospitalised following a manic relapse. We investigated how activity data might be used to develop an early warning system for manic and other types of episodes in people diagnosed with bipolar disorder.

Complexity can be defined in terms of the predictability of a sequence of observations of the same phenomenon, such as activity recorded during a two-minute observation period. Throughout any 24-hr period, the activity level fluctuates from one two-minute epoch to the next, the extent of these fluctuations providing information about the complexity of the associated psychomotor processes. Regular fluctuations indicate a low level of complexity, the least complex example being the regular tick of a clock. As a process becomes more complex its fluctuations become more erratic, an extreme example being random noise. Most physiological systems exhibit behavior somewhere between these two extremes. Importantly, research in several domains has found that it is worthwhile monitoring changes in complexity as significant decreases may suggest the onset of pathology (Heath, 2004).

Some medical disorders are characterized by a reduction in the complexity of at least one associated measurement, such as heart rate in the case of heart dysfunction, EEG amplitude and frequency for epileptic seizures, and mood rating variability for people with 
unipolar depression (see Heath, 2004, for further examples). In epileptic seizures, for example, the pre-ictic period prior to the seizure onset is characterized by an increase in slowwave activity detectable as a reduction in complexity of the corresponding EEG waveform. This change can be observed by sensitive measures that make full use of the temporal relationships between successive waveform measurements (Li, Yan, Liu \& Ouyang, 2014). When a significant change has been detected, a warning signal may be presented to the person, and procedures adopted to avoid a fully expressed epileptic seizure (Santaniello, Burns, Golby, Singer, Anderson \& Sarma, 2011). We propose that a similar diagnostic signal might be used to detect the imminent onset of a manic episode in people diagnosed with bipolar disorder.

\section{Entropy}

Entropy is commonly defined as the amount of disorder in a dynamical system. As large values of entropy reflect an increase in the complexity of the underlying dynamic process, quantitative entropy indices serve as useful proxies for complexity in physiological and behavioral systems. Here, we focus on two measures of entropy, Sample Entropy and Permutation Entropy. Sample Entropy is a popular index for estimating the complexity of a sequence of observations (Richman \& Moorman, 2000). The idea behind Sample Entropy is not too complicated. A small set of time series observations are considered within a data window. This window is then moved along one observation at a time and the contents of these successive windows are compared. In a regular series with low entropy, there will be little change in the numbers from one window to the next. However, if the numbers in one window deviate from those observed in the immediately preceding window, a larger entropy measure will be obtained. Sample Entropy measures the extent to which a time series is irregular in its observation sequence, being low for a regular series that hardly changes, and large for an irregular series such as white noise. Prior to conducting a Sample Entropy 
analysis, the data are transformed by subtracting the mean and dividing by the standard deviation to obtain a series with zero mean and a standard deviation equal to 1 (see the Appendix for details).

Although Sample Entropy has been used to analyse activity data (Tahmasian, Khazaie, Golshani \& Avis, 2013), it may have limited value because in a study that used activity measures to compare controls with patients hospitalised for depressive and manic episodes, none of the inter-group differences in Sample Entropy was statistically significant (KraneGartiser, Henriksen, Morken, Vaaler \& Fasmer, 2014, Table 4). Although we evaluate the use of Sample Entropy in this paper, another interesting entropy measure, Permutation Entropy, will also be considered. All calculations were performed using programs written in the $R$ Statistical Programming Language (R Core Team, 2021).

\section{Permutation Entropy as a Complexity Index}

Permutation Entropy (PE), a technology devised by physicists (Bandt \& Pompe, 2002, Keller

\& Sinn, 2005), has been successful in predicting the onset of epileptic seizures (Li, Ouyang \& Richards, 2007) and in monitoring vital signs during anaesthesia (Olofsen, Sleigh \& Dahan, 2008). Within each data set, all possible orders of the numbers contained in short successive data sequences (data windows), say three successive observations, are counted and a frequency distribution obtained. This distribution is compared with the one obtained when all possible orders within the small data sequence are equally likely (six possible orders each with probability $1 / 6$ when there are three successive observations in a data window). The resulting PE estimate stays the same whenever any data transformation that preserves the order of the numbers in each small data sequence is applied. Such data transformations include adding 10 to every number, multiplying every number by 10 or squaring every number in the sequence. The PE estimate can be computed over successive data windows, thus allowing change in entropy over time to be monitored, as is necessary in real-time 
biomedical applications. Simulations have shown that the Permutation Entropy estimate is less affected by measurement noise than some other complexity measures. Permutation Entropy ranges between 0 and 1, the larger the value the greater the complexity. It can be more sensitive to change than other complexity measures, such as Sample Entropy.

Before computing PE for a data sequence, three parameters must be selected, the permutation length, $d$, the time-series lag, $L$, and the window width, $w . L$ can be set to 1 by summing data values over successive observations, say five, to produce total activity in every ten-minute period. This ensures that the correlation between successive ten-minute periods, that is the autocorrelation at increasing delays between the numbers, is sufficiently small. This procedure was adopted in the present analyses in which the original activity data was recorded every two minutes. The permutation length, $d$, is set to a small integer between 5 and 7 so that the number of possible permutations within a data window is not too large. A range of permutations between $5 !=120$ and $7 !=5040$ is considered satisfactory (Cao, Tung, Gao, Protopopescu \& Hively, 2004). The window width, $w$, can be set to an arbitrary number that provides a satisfactory trade-off between the relative frequencies of the orders within a window and the rate of change in the complexity of the time-series. However, it is convenient to set $w$ equal to the number of possible permutations of $d$ objects where $d$ is the sequence length. In the analyses to follow, $d=6$ and $w=6 !=720$. The method for estimating Permutation Entropy is contained in the Appendix.

\section{Detecting Change in a Time Series Using an Adaptive Kalman Filter}

In patients with a chronic illness, such as bipolar disorder type I, it is important to have some quantitative indicator of change in their health status, such as their current mood. Based on evidence that activity changes in bipolar disorder can be diagnostic of mood state (Bullock \& Murray, 2014), a mood change detection strategy using a complexity measure derived from activity data, such as Permutation Entropy, is worth considering. One possible methodology 
detects changes in the temporal properties of $P E$ by predicting future observations using previous observations from the same series.

In its simplest form, we compute a linear autoregressive model of order $m$ that predicts the $n$-th value in a series from a weighted combination of the previous $m$ observations (see the Appendix for more details about autoregressive models). If this model fits the baseline data reasonably well and there is no change over time in the prediction equation parameters, the prediction errors will be small and randomly distributed around a mean of zero. However, any change in the underlying mood control system may result in an increase in prediction error as the current prediction model becomes inadequate. To better track observations, the prediction equation parameters are adjusted to minimise future prediction errors. The accumulated prediction error, measured during the change and standardised by dividing by the best estimate of the standard deviation of these errors, provides information about a possible modification of the underlying psychomotor control process. This may result from a change in mood or more generally a change in the person's ability to cope with environmental demands.

This change detection technology is known as the Adaptive Kalman Filter, a general method used by engineers to control systems perturbed by environmental influences, one example being autopilot control of an aircraft during atmospheric turbulence. The mathematical details of the method are contained in Heath (2000, pp.37-43). A summary and a simplified example calculation are contained in the Appendix.

\section{Using Sample Entropy to Detect Change in Activity in a Person Susceptible to Manic Episodes}

Changes in activity over time in people diagnosed with bipolar disorder are suggested by the recurrent fluctuations in mood from depression, frequently accompanied by low levels of 
activity, through periods of euthymia and mania that are typically accompanied by increases in activity level (Goodwin \& Jamison, 2007). As indicated in a previous section, actigraphy, which is commonly used to measure sleep and circadian rhythms under naturalistic conditions, records three-dimensional movement using an accelerometer and on-board memory storage (Ancoli-Israel, Cole, Alessi, Chambers, Moorcroft \& Pollak, 2003).

The actigraphy data analysed in this section have been described in a case report (Bullock, Judd \& Murray, 2014) that used activity measurements collected by a wrist-worn ActiGraph to monitor the behaviour of a 20 -year-old male diagnosed with bipolar disorder type I. The participant was enrolled in a 12-month prospective study of actigraphicallymeasured sleep and circadian function as predictors of daily mood in bipolar disorder. For this patient, activity was recorded for 103 days prior to their withdrawal from the study due to hospitalisation for a manic episode.

The raw ActiGraph data obtained two weeks prior to this patient's hospital admission showed that there was reasonably normal activity and sleep patterns for the first eight days, followed by the sudden appearance of severely disrupted sleep and an increase in activity in the five days prior to hospitalisation (Bullock, Judd \& Murray, 2014). This increase in activity and sleep disruption is shown in the bottom graph of Figure 3, using data recorded on day 120 , just prior to the hospital admission. The sleep pattern changed to a 48 -hour cycle and average activity levels increased markedly.

Before computing Sample Entropy using the activity data, the log-log spectrum, the relationship between the logarithms of the spectral power and the frequency, was computed for data obtained for each two-minute period on each of the 103 days. The slope of the loglog spectrum provides information about temporal properties of the data, including the possibility of long-term persistence in the process responsible for generating activity data. If this occurs, the person's current activity state is likely to last a long time. 
Figures 1 to 3 show the original activity data, the power spectrum, and the log-log spectrum, recorded on Day 30 during what appears to be a euthymic period, Day 81, when the Permutation Entropy analyses below suggested an early morning change in complexity, and on Day 102, just before the participant was hospitalised. The best-fitting linear fit to the $\log -\log$ spectrum provided slope estimates of $-1.21,-0.88$ and -1.03 , for days 30,81 and 102 , respectively.

The slope estimates for all 103 days are plotted in Figure 4. The log-log, or $1 / f$, spectral slope fluctuated over days, $M=-1.07, S D=.20$, as shown by the dotted $95 \%$ confidence lines shown in the Figure. The mean slope was significantly less than $-1.0, t(102)=3.81$, $p<.001$. The only autocorrelation observed in the slope measures over days was a small significant positive correlation of $0.23(p<.05)$ for observations five days apart. Thus, these linear measures provide minimal evidence of an imminent manic episode necessitating hospitalisation on Day 103, except possibly for a large negative slope on Day 91 that exceeds the confidence bounds. It is worth noting that Gottschalk, Bauer \& Whybrow (1995) observed a more negative $1 / f$ slope for bipolar patients when compared with controls. However, this has not been a general finding (Heath, Heiby \& Pagano, 2007). Although slopes close to -1 suggest long-term correlations in the daily data sequences, often associated with a complex underlying process, a more detailed analysis of the activity data would be required to determine its practical value.

Whenever there is evidence suggesting that a linear autocorrelation or spectral model for time series data may not be appropriate, it is worthwhile investigating alternative methods for estimating the complexity or information content of the data. A preliminary Sample Entropy analysis was computed for the activity data obtained from this patient. The analysis, which used a time series matching window, $m=2$, and a critical distance, $r=0.15$, compared the similarity of results obtained when the size of the matching window was increased to 3 
using the same critical distance. As shown in the Appendix, Sample Entropy is computed by comparing the number of subseries separated by a vector distance less than 0.15 with the distances between all possible pairs of data vectors using window sizes of 2 and 3 , this value being 24 .
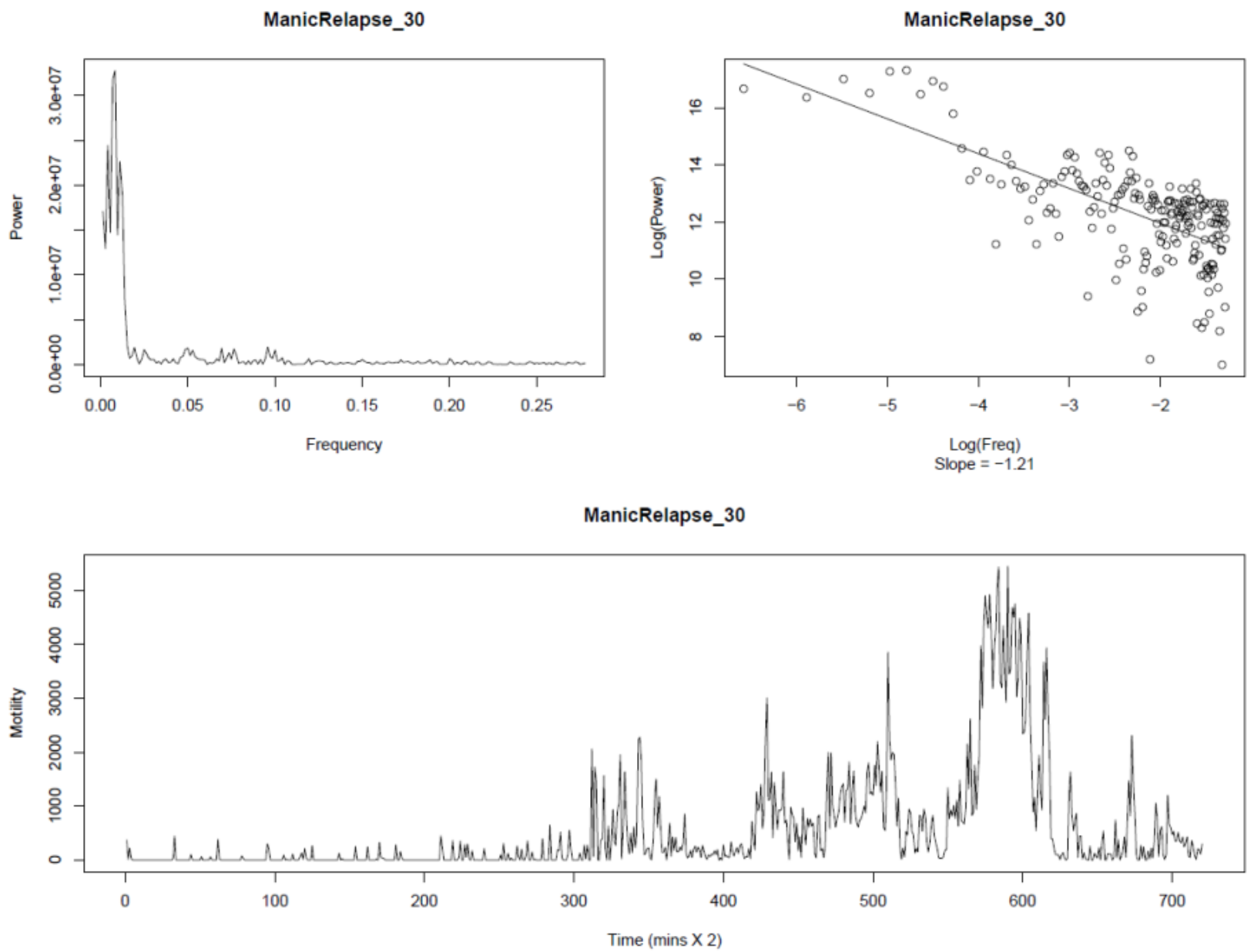

Figure 1. Activity data for the bipolar client on day 30 . The bottom graph shows the activity, or motility, recorded every two minutes. The upper graphs show the power spectrum (left) and the log-log spectrum with its line of best fit (right).

An interesting finding from the Sample Entropy analysis was a sudden increase in the variability of the entropy values in the last few days prior to hospitalisation, but only for activity data recorded between the morning hours of 0400 and 0800 . This result is consistent with the phase shift in sleep onset time and possibly the lack of sleep on alternate days shown in Figure 1 of Bullock, Judd and Murray (2014). Figure 5 exaggerates this effect by plotting 
the ratio of Sample Entropy for activity recorded during (0400-0800) compared with the average Sample Entropy for later periods in the day (0800-2400).
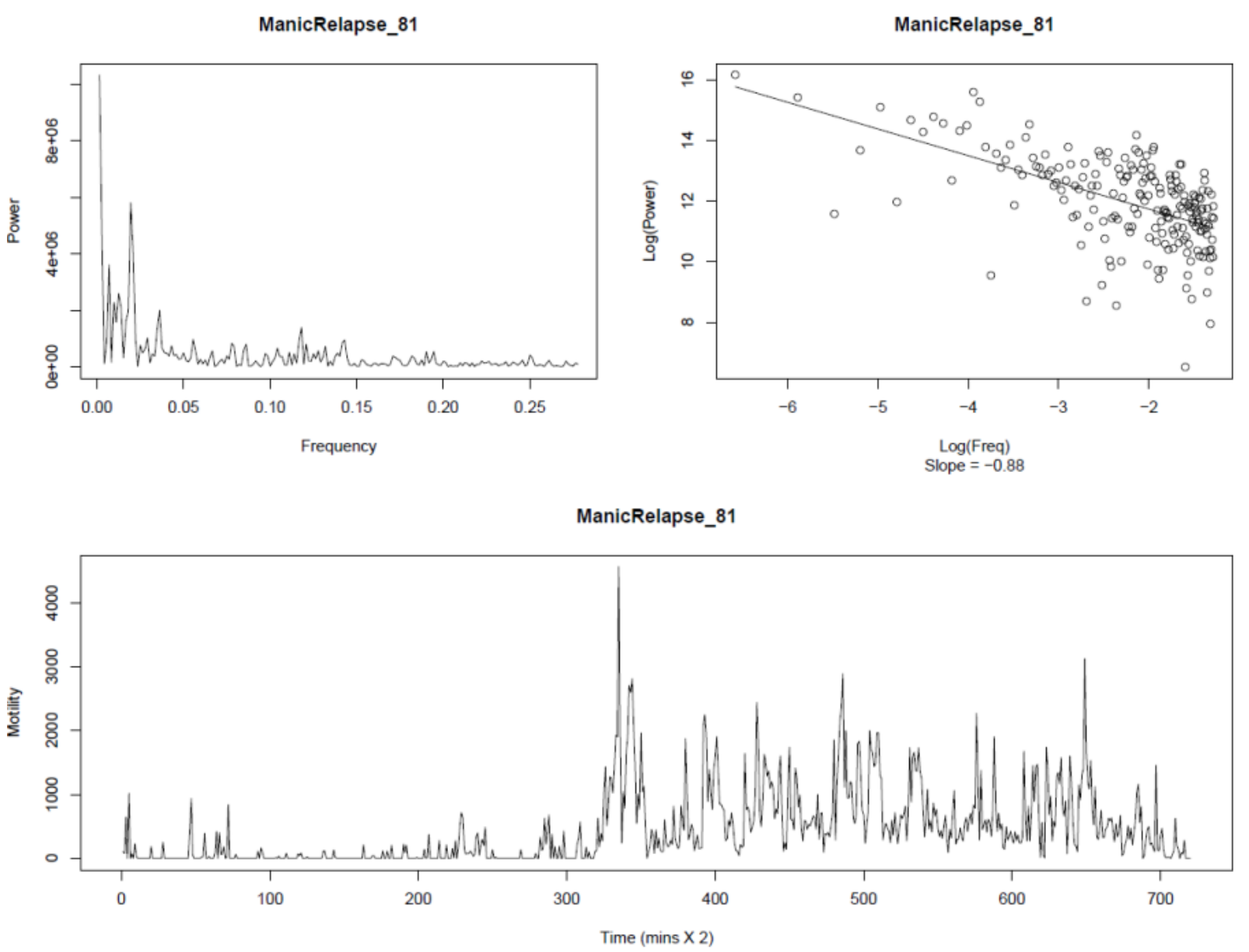

Figure 2. Activity data for the bipolar client on day 81 . The bottom graph shows the activity, or motility, recorded every two minutes. The upper graphs show the power spectrum (left) and the log-log spectrum with its line of best fit (right).

Based on similar findings from the Permutation Entropy analysis to follow, diurnal changes in Sample Entropy were estimated by computing the ratio of average Sample Entropy during the evenings (2000-0800) and average Sample Entropy during the day (08002000). Figure 6 shows a rapid and large decrease in this ratio between days 70 and 85 followed by an increase in the ratio thereafter. This turning point in the Sample Entropy ratio graph occurred almost three weeks before the patient needed to be admitted to hospital. 

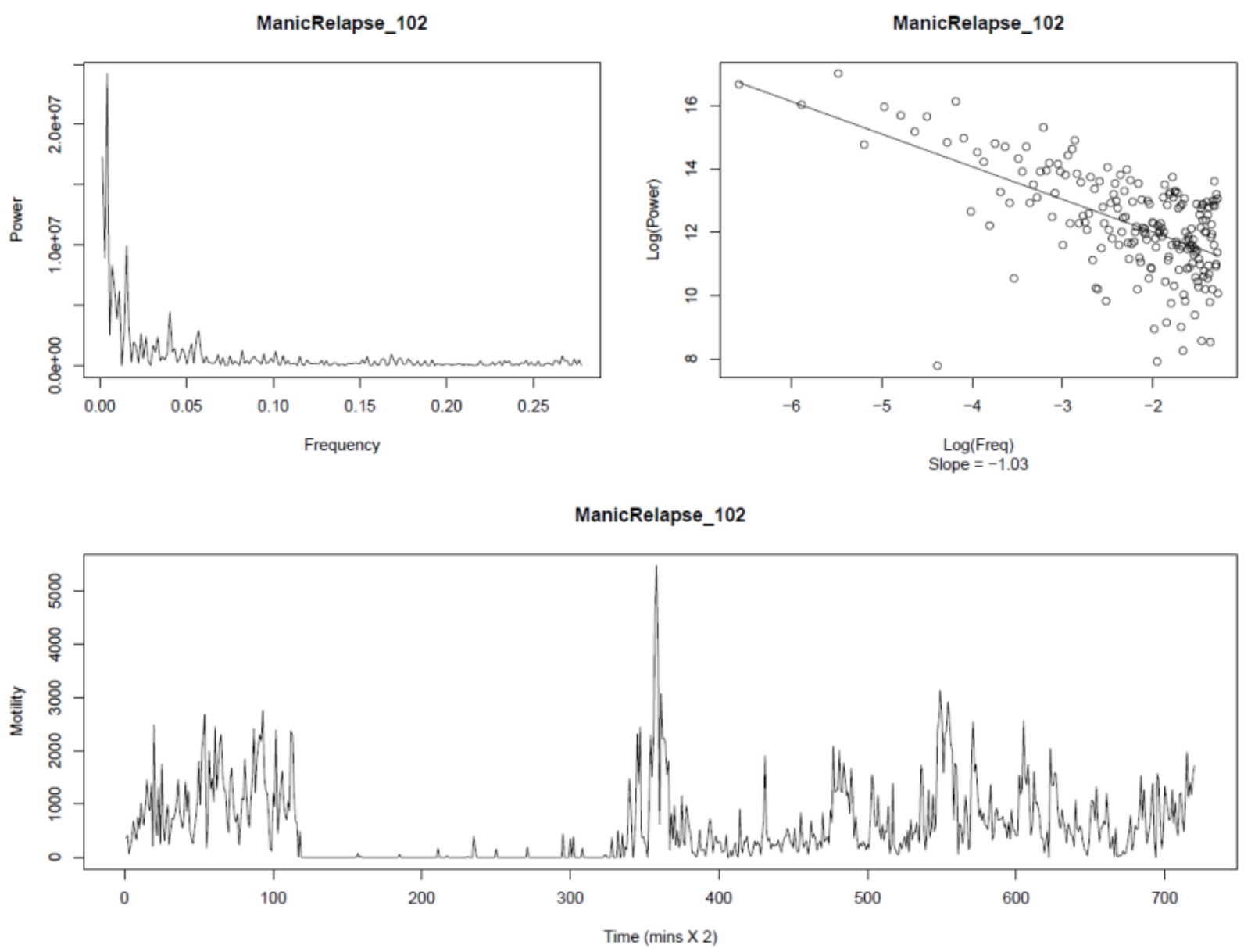

Figure 3. Activity data for the bipolar client on day 102, a few days before hospital was required for a serious manic relapse. The bottom graph shows the activity, or motility, recorded every two minutes. The upper graphs show the power spectrum (left) and the log-log spectrum with its line of best fit (right).

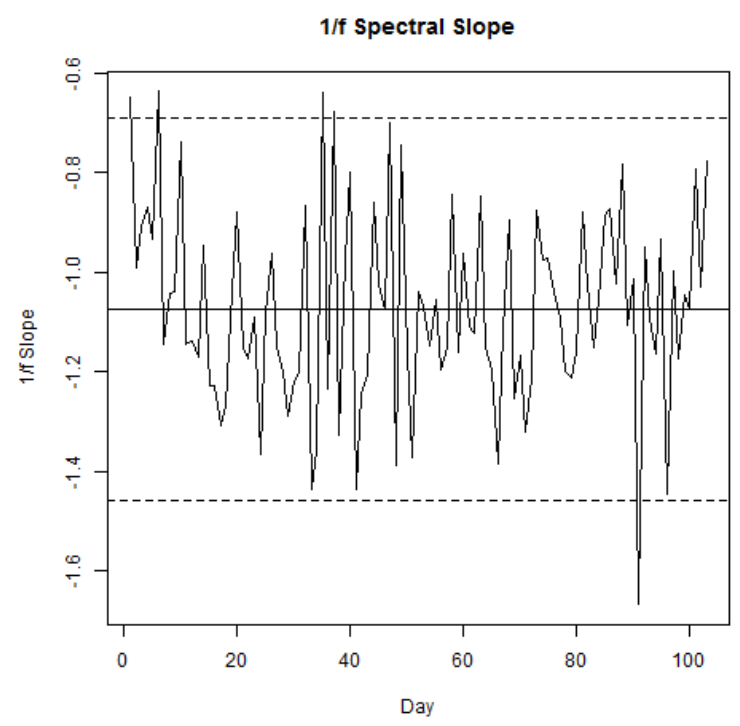

Figure 4. The $1 / f$ spectral slope for each day's activity for the Bipolar I patient using 720 cumulated activity measures recorded every two minutes. The dotted lines show the $95 \%$ confidence interval about the mean $1 / f$ slope (solid line). 


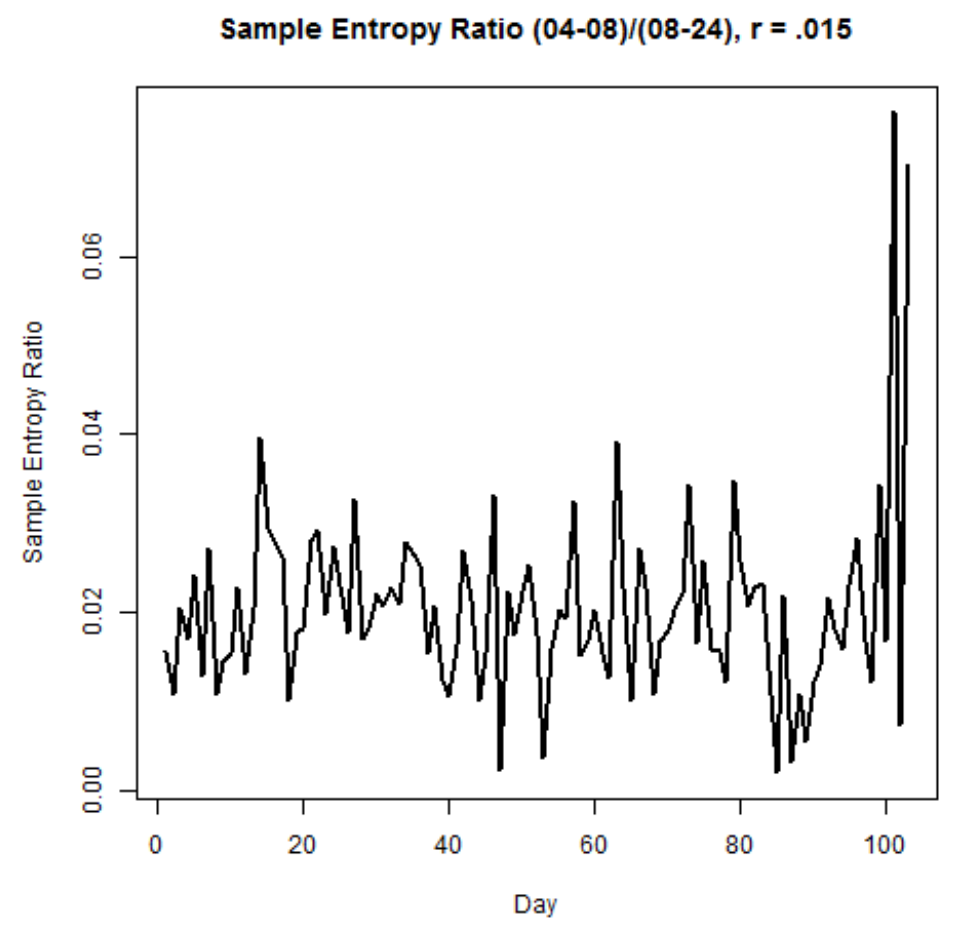

Figure 5. Sample Entropy ratio comparing Sample Entropy between 4 am and 8 am and the rest of the day ( 8 am to midnight) for each day of the activity recording for the Bipolar I client ( $m=2, r=0.15)$.

Sample Entropy Ratio Night/Day, $r=.015$

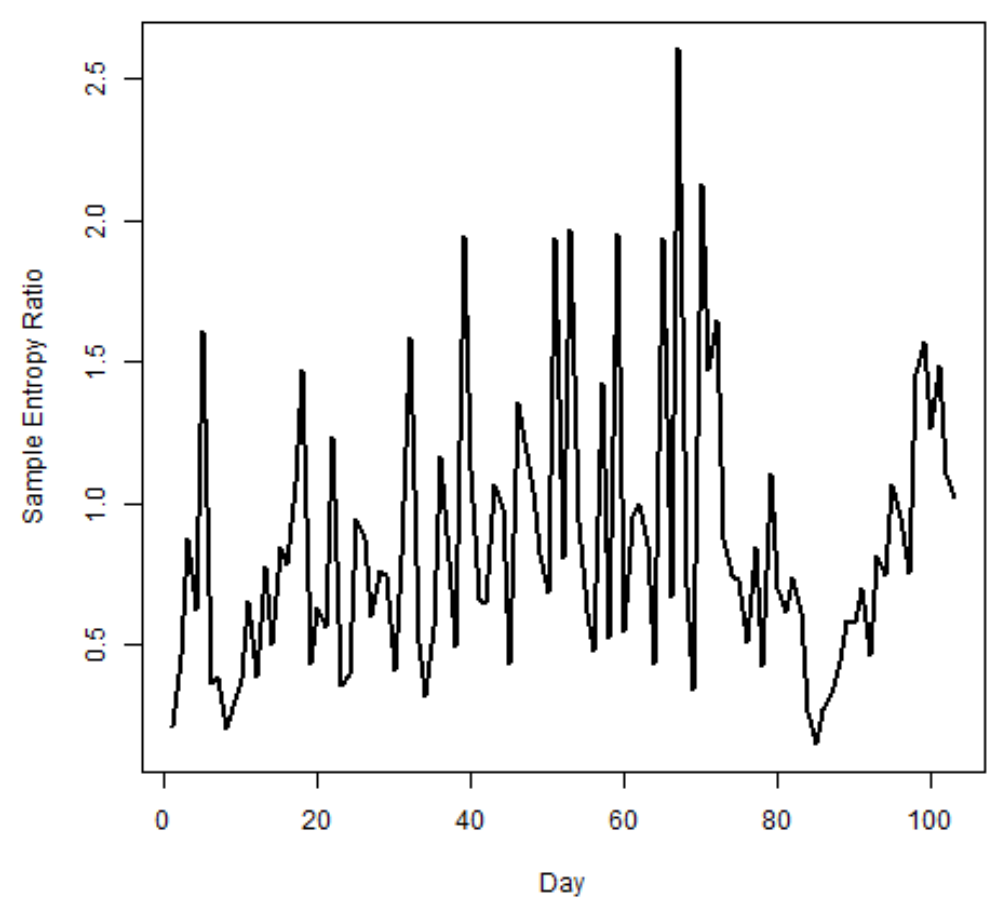

Figure 6. Sample Entropy ratio comparing Sample Entropy between 8 pm and 8 am (Night) and between 8 am and $8 \mathrm{pm}$ (Day) for each day of the activity recording for the Bipolar I client $(m=2, r=0.15)$. 
The presence of a signal indicating a twice-daily variation in Sample Entropy suggests the presence of an underlying circadian component to the complex system disturbance observed in mood disorder (Indic et al., 2001; Murray, 2007; Murray, Allen, Trinder \& Burgess, 2002). This idea is also supported by animal circadian rhythm data in which the circadian system plays a key role in the complex systems behaviour as measured by activity data (Hu, Scheer, Ivanov, Buijs \& Shea, 2007). Although this analysis using Sample Entropy might have alerted the patient and his medical team to an impending relapse, it may not have provided sufficient warning to prevent hospitalisation. For this worthwhile purpose, we now consider how Permutation Entropy can be used to provide a warning of a possible future serious manic (or depressive) episode.

\section{An Application of Permutation Entropy for a Single Case}

Permutation Entropy $(P E)$ values can be accumulated over temporal windows to detect a significant change in complexity that might suggest deterioration in a medical condition; a significant increase in $P E$ might indicate improvement. Medical applications of $P E$ are based on the idea that disease can be detected by a decrease in the estimated complexity of physiological data. Perhaps good health can be best considered in terms of an optimal value of physiological and/or behavioural complexity. For example, in Figure 1 from Li, Ouyang \& Richards (2007), $P E$ detected the early signs of a looming epileptic seizure before its later detection using Sample Entropy.

To estimate $P E$, activity data were accumulated over five successive observation periods to provide total activity recorded during each ten-minute period. These data were incremented by 1 to ensure that there were no zero values, and a logarithmic transformation was applied. The resulting data series was then differenced to remove any linear trend. $P E$ was computed separately for data obtained by concatenating observations from each four- 
hour period (viz., 0000-0400, 0400-0800, 0800-1200, 1200-1600, 1600-2000 and 20002400) over each of the 103 days. Each of these six data sets contained 2471 observations. For each four-hour period, the data window, $w$, contained $6 !=720$ observations, the dimension was set at $d=6$ and the lag $L=1$. The first $P E$ estimate was identified with a time located at the end of the first window, implying that the $P E$ estimates started 720 ten-minute periods after the beginning of the series on Day 6 . This procedure was necessary to ensure that successive observations were relatively uncorrelated and there was sufficient order information within a data window to provide accurate $P E$ estimates. The logistic transform of $P E, \log (P E /(1-P E))$ was used in the following plots.

The results of the $P E$ analyses for each time period are depicted in Figures 8 to 12 . Each figure contains three graphs, the original activity data that was pooled over 10 minutes, log-transformed and differenced, a logistic transformation of $P E$ estimates, and the summed standardised residuals estimated by applying the Adaptive Kalman Filter to the original $P E$ estimates. In each time period, the autoregression model employed by the Adaptive Kalman Filter was the one that best fit the data. For example, the "Model = AR(6)" label in the bottom graph of Figure 7 indicates that the current $P E$ value was predicted most accurately using the previous 6 values.

The top graphs in each Figure show the variability of the transformed activity data was relatively high in the morning hours, $00-08$, but much less for the rest of the day. The intermittent nature of the data, indicated by periods of sharp spikes of varying duration, depends on the time of day, being most prominent before noon on several days. Otherwise, there is little information in the transformed activity data itself that might be used to predict the mania episode that required hospitalisation on day 104. 
Time Period $=0->4$ Pooled over 5 two minute activity measures

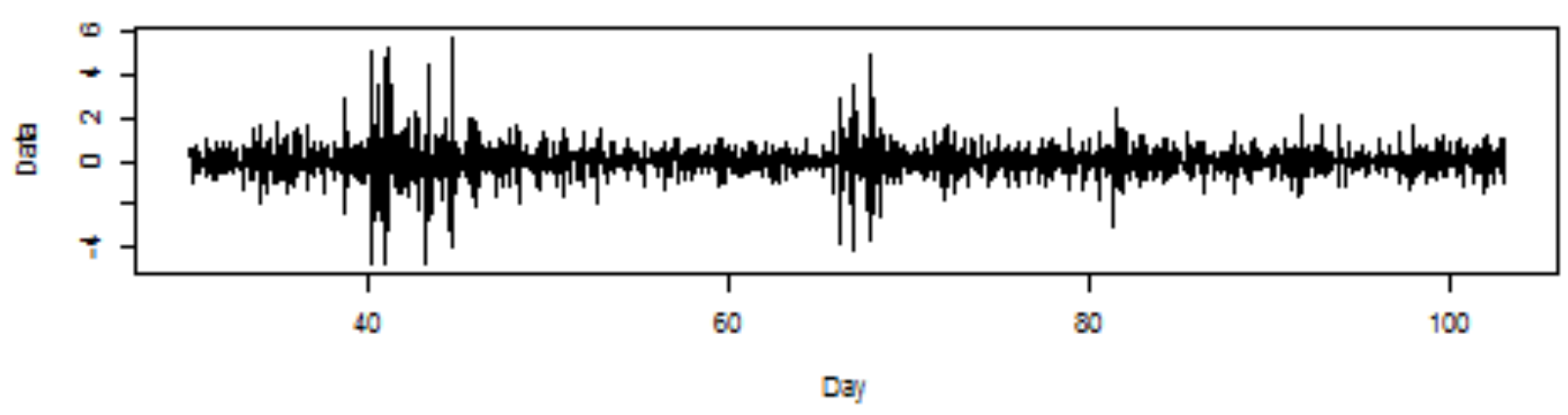

Permutation Entropy, $d=6$
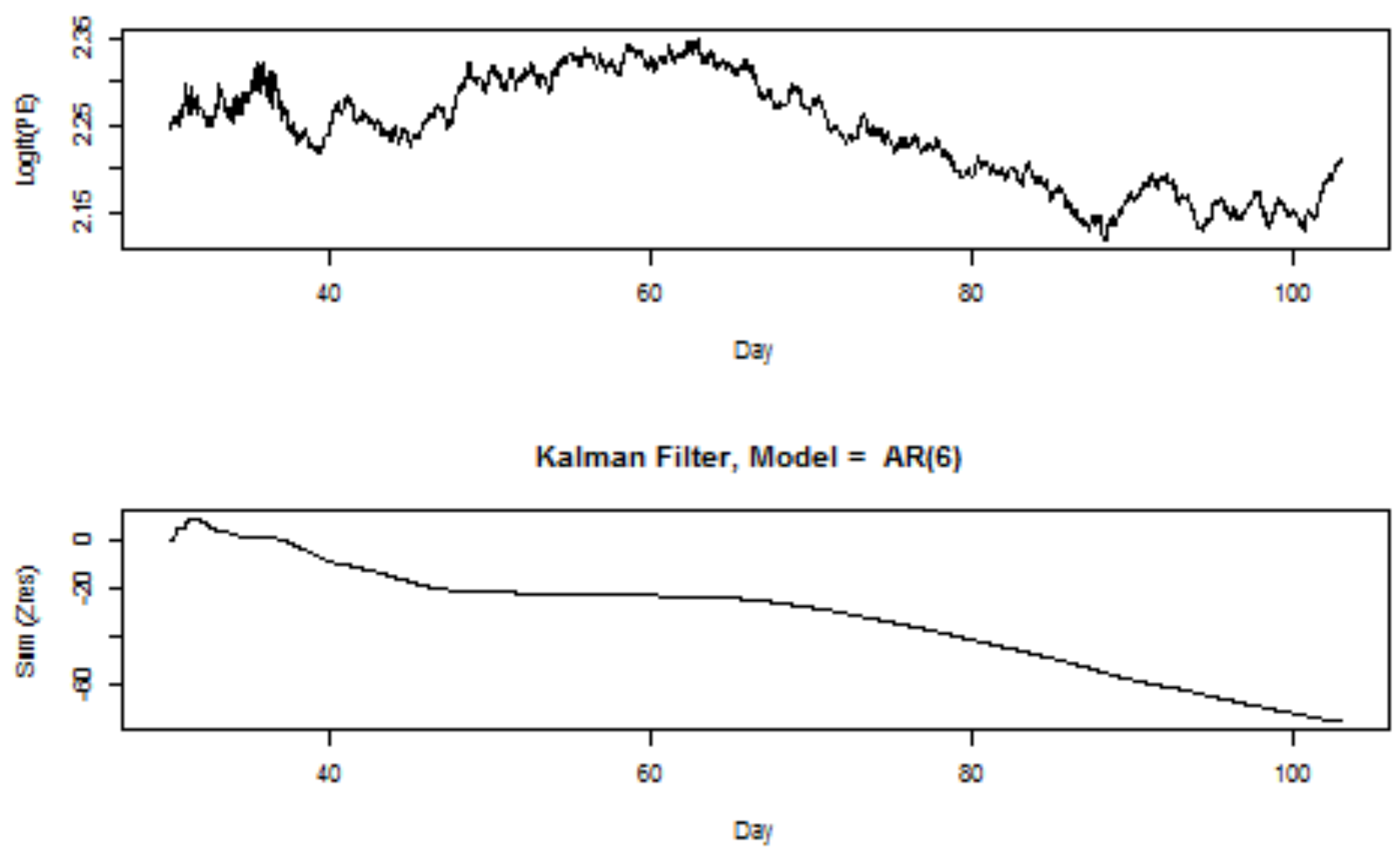

Figure 7. Changes in activity recorded between midnight and 4 am on 103 successive days for a young male hospitalised for a manic episode on day 104. The top graph shows difference data obtained by a logarithmic transformation of original activity data pooled over five successive observations (10 mins). The middle graph shows changes over time in the logistic transformation of Permutation Entropy estimates. The bottom graph shows the accumulated standardized residuals computed using an Adaptive Kalman Filter with an autoregression equation of order 6 . 
Time Period = 4-->8 Pooled over 5 two minute activity measures

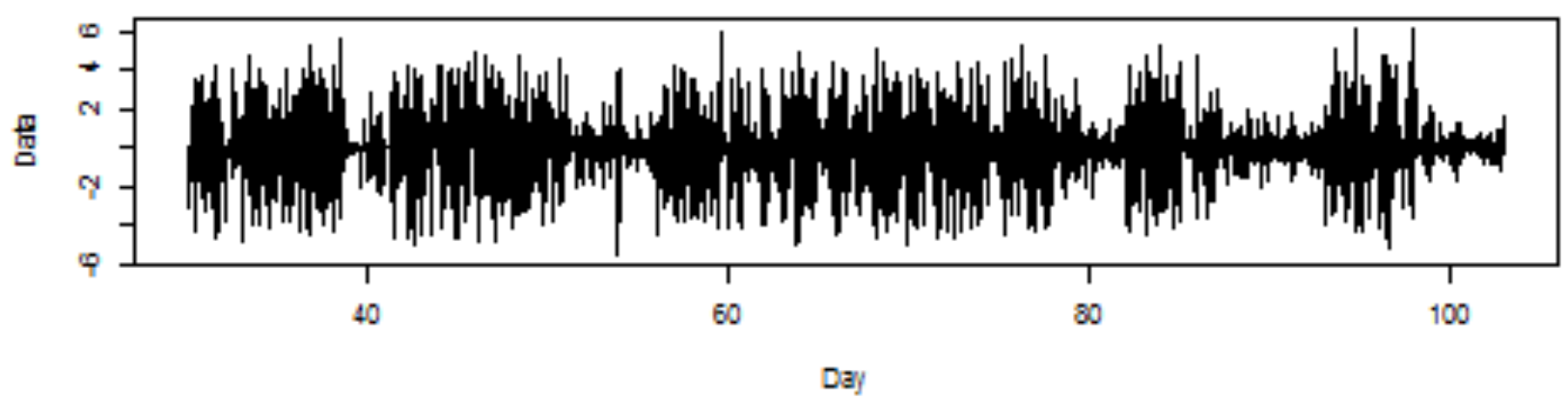

Permutation Entropy, $\mathrm{d}=6$
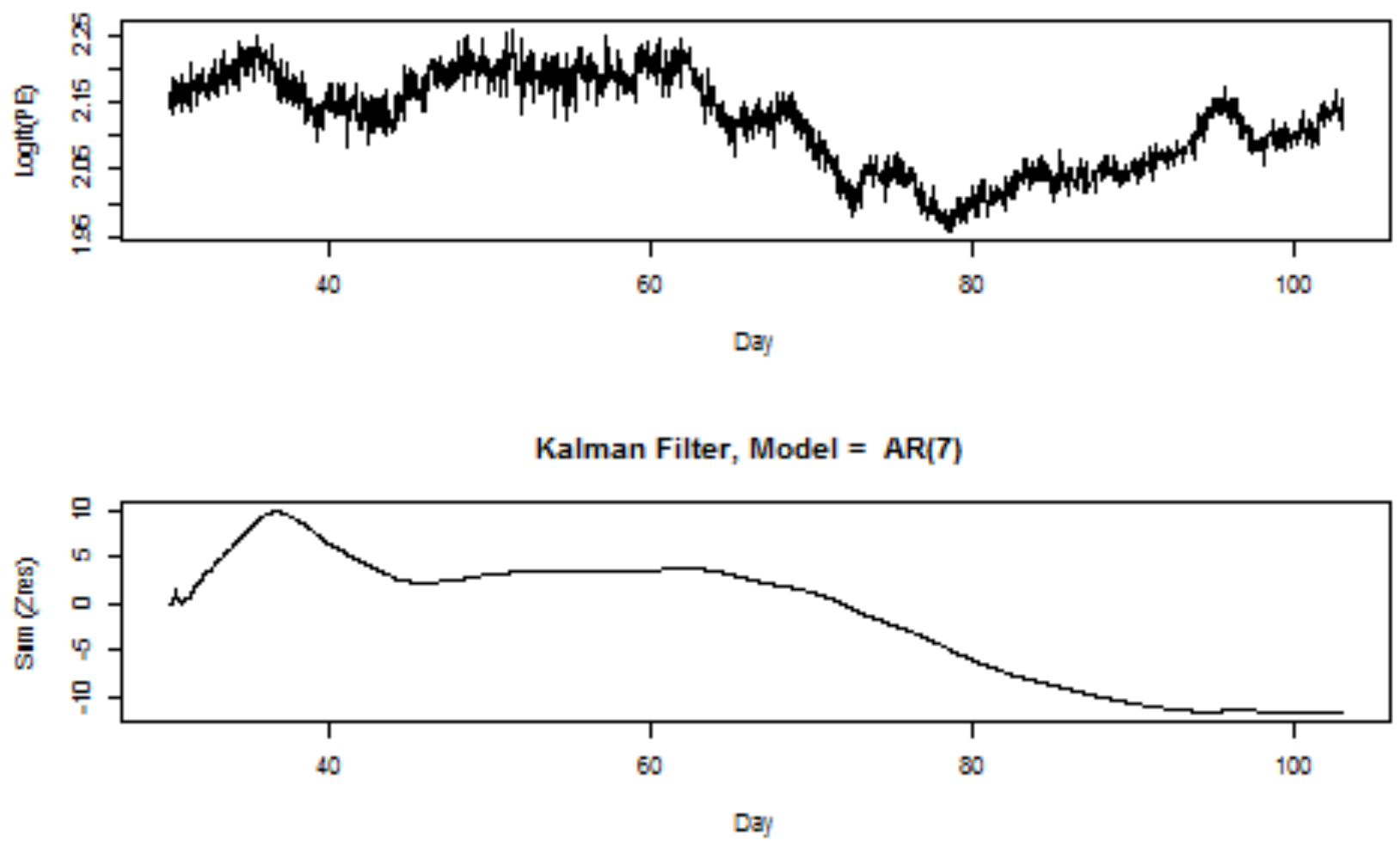

Figure 8. Changes in activity recorded between 4 am and 8 am on 103 successive days for a young male hospitalised for a manic episode on day 104. The top graph shows difference data obtained by a logarithmic transformation of original activity data pooled over five successive observations (10 mins). The middle graph shows changes over time in the logistic transformation of Permutation Entropy estimates. The bottom graph shows the accumulated standardized residuals computed using an Adaptive Kalman Filter with an autoregression equation of order 7 . 
Time Period = 8-->12 Pooled over 5 two minute activity measures

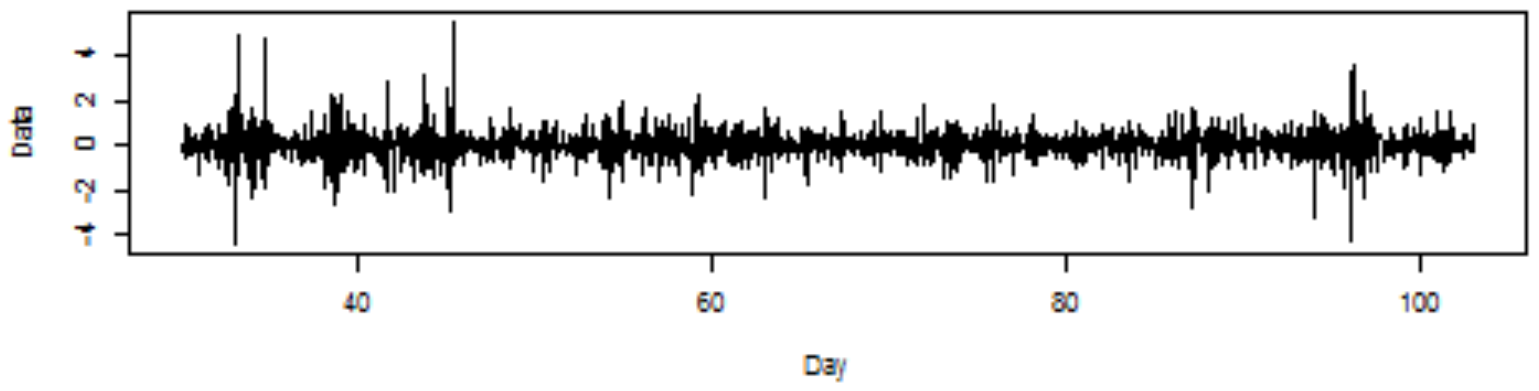

Permutation Entropy, $d=6$
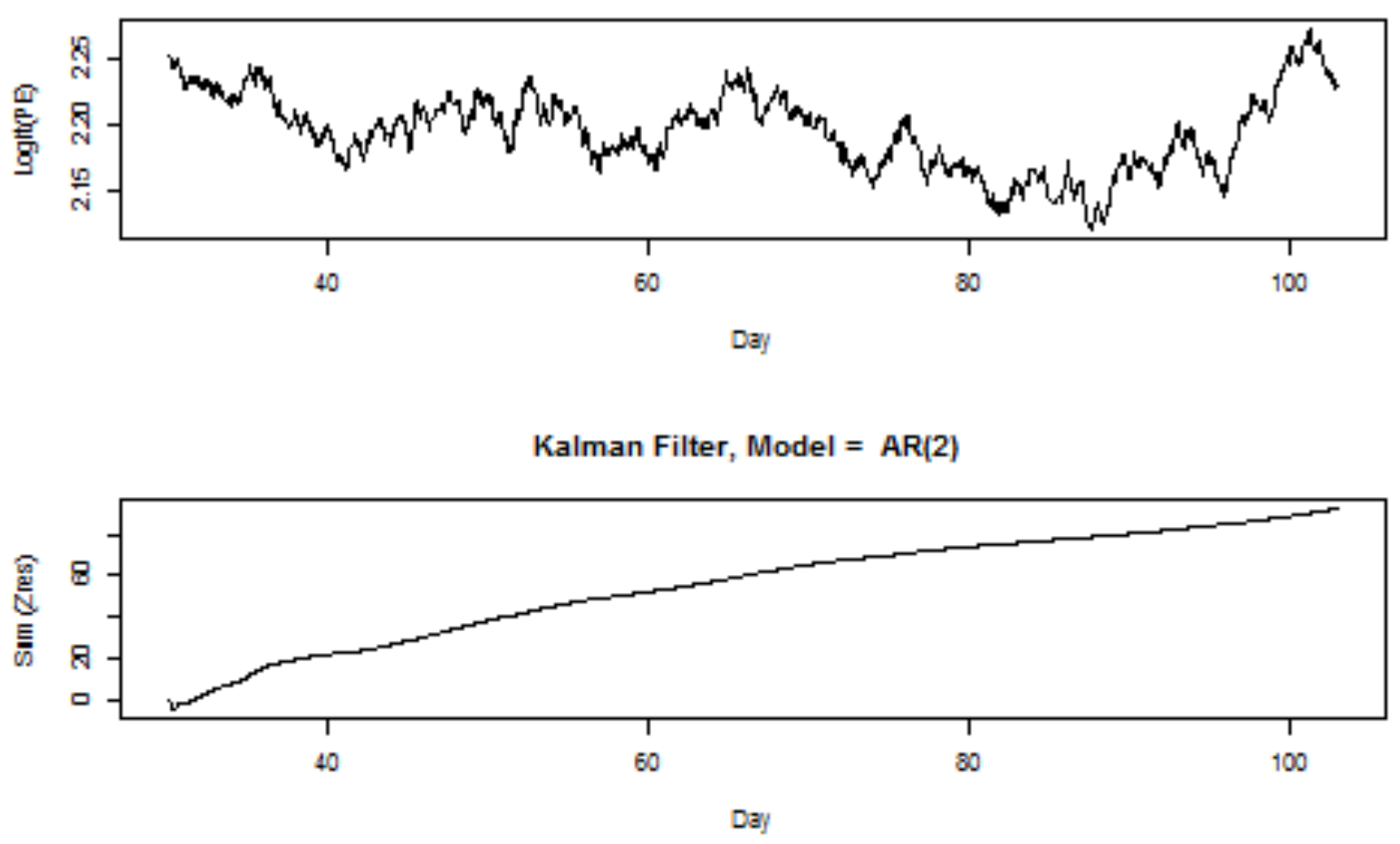

Figure 9. Changes in activity recorded between 8 am and midday on 103 successive days for a young male hospitalised for a manic episode on day 104 . The top graph shows difference data obtained by a logarithmic transformation of original activity data pooled over five successive observations (10 mins). The middle graph shows changes over time in the logistic transformation of Permutation Entropy estimates. The bottom graph shows the accumulated standardized residuals computed using an Adaptive Kalman Filter with an autoregression equation of order 2 . 
Time Period = 12->16 Pooled over 5 two minute activity measures

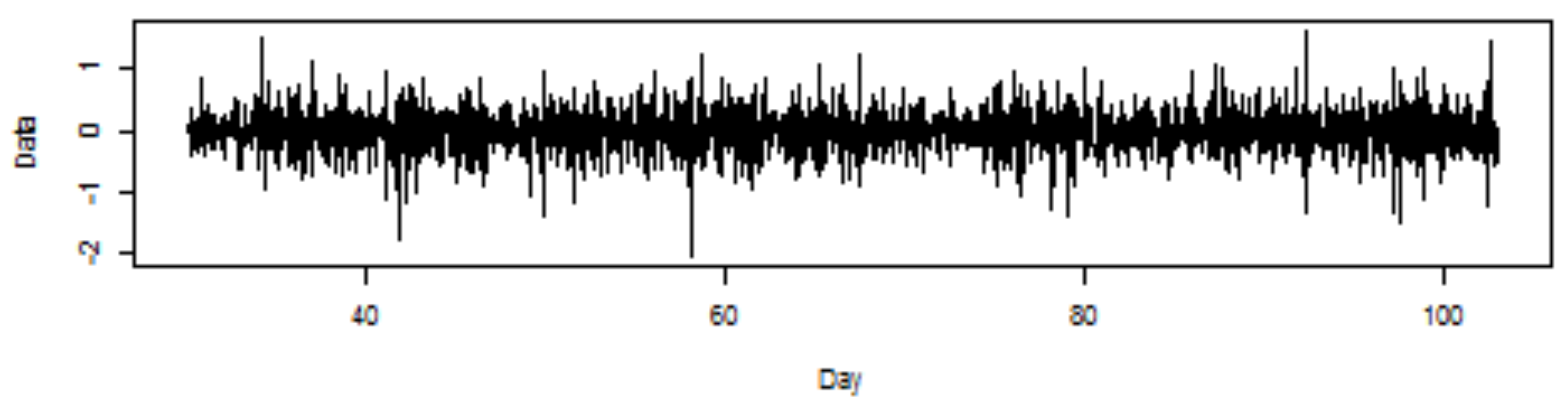

Permutation Entropy, $d=6$

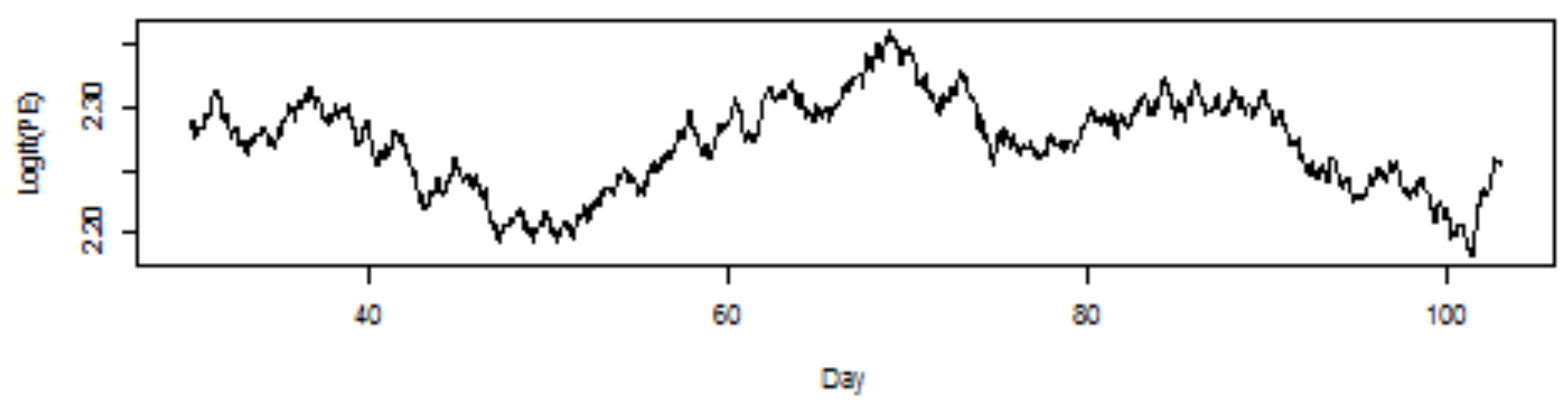

Kalman Filter, Model $=\operatorname{AR}(2)$

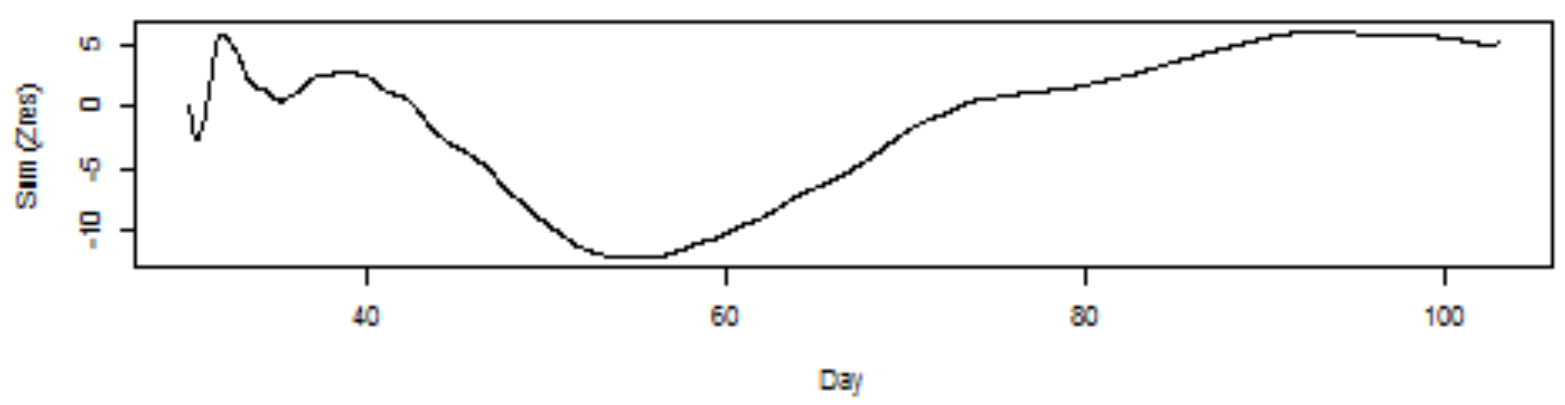

Figure 10. Changes in activity recorded between midday and $4 \mathrm{pm}$ on 103 successive days for a young male hospitalised for a manic episode on day 104. The top graph shows difference data obtained by a logarithmic transformation of original activity data pooled over five successive observations (10 mins). The middle graph shows changes over time in the logistic transformation of Permutation Entropy estimates. The bottom graph shows the accumulated standardized residuals computed using an Adaptive Kalman Filter with an autoregression equation of order 2. 


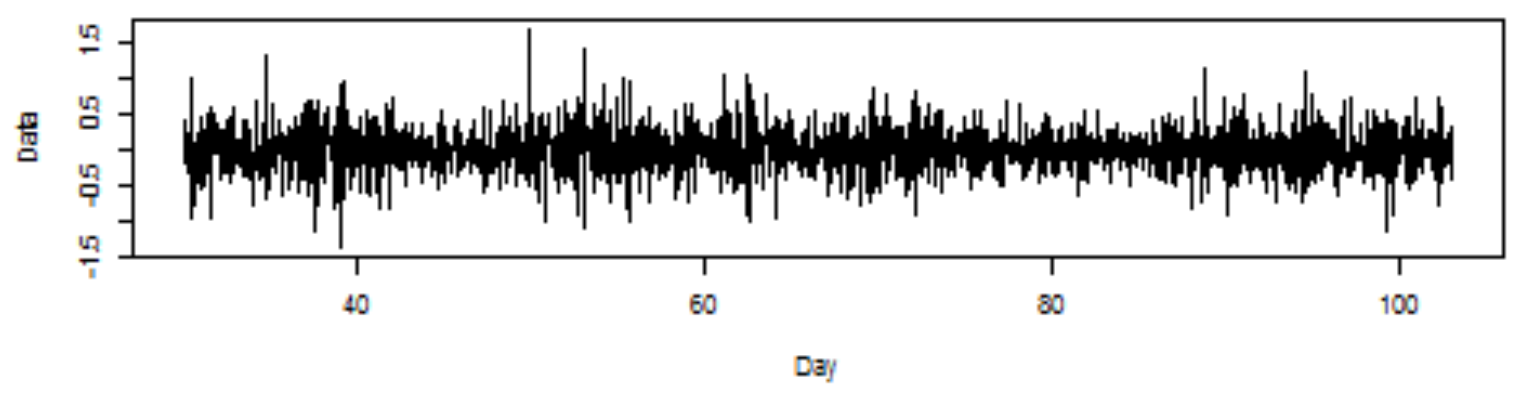

Permutation Entropy, $d=6$

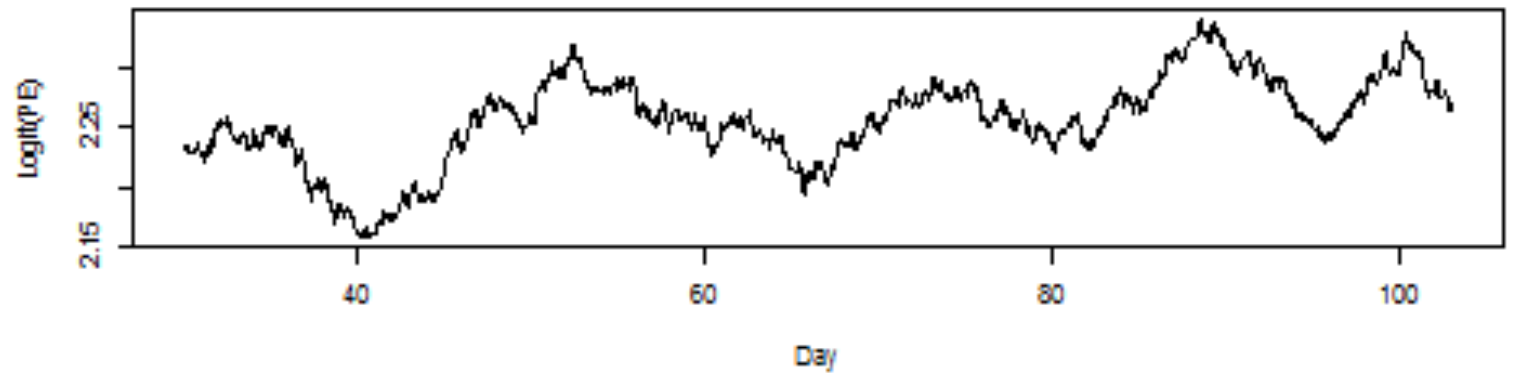

Kalman Filter, Model $=$ AR(2)

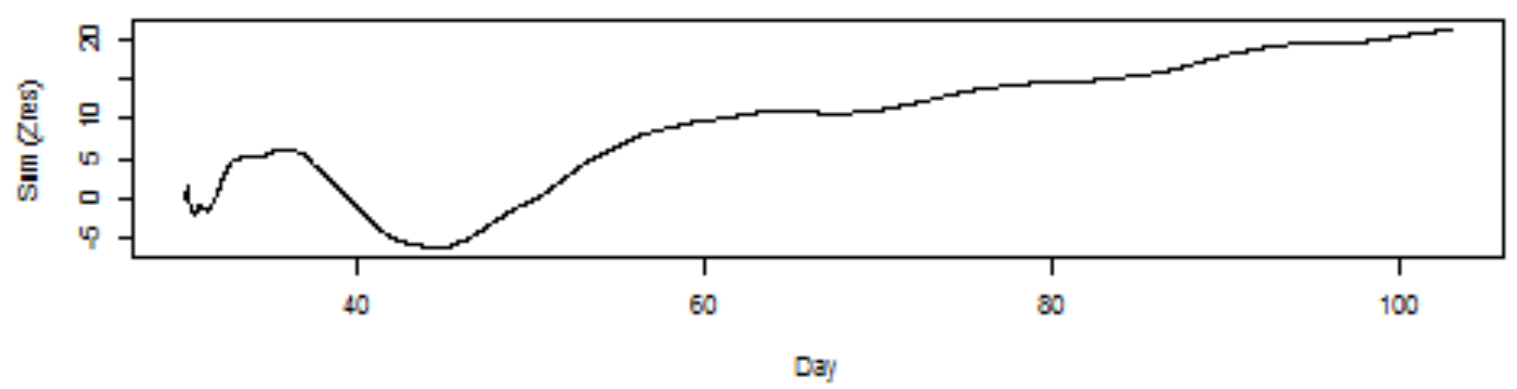

Figure 11. Changes in activity recorded between $4 \mathrm{pm}$ and $8 \mathrm{pm}$ on 103 successive days for a young male hospitalised for a manic episode on day 104. The top graph shows difference data obtained by a logarithmic transformation of original activity data pooled over five successive observations (10 mins). The middle graph shows changes over time in the logistic transformation of Permutation Entropy estimates computed. The bottom graph shows the accumulated standardized residuals computed using an Adaptive Kalman Filter with an autoregression equation of order 2 . 
Time Period $=20->24$ Pooled over 5 two minute activity measures

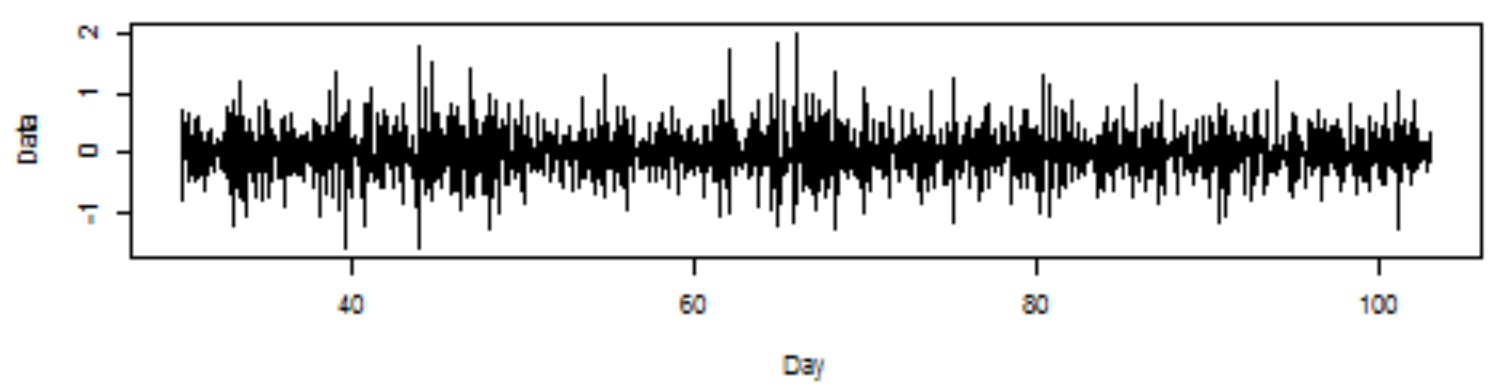

Permutation Entropy, $d=6$

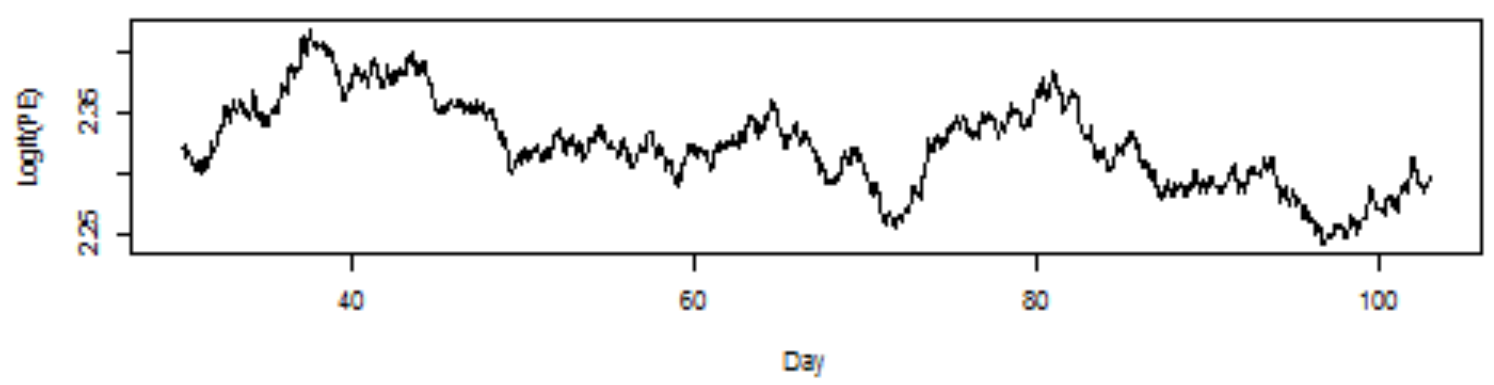

Kalman Filter, Model $=$ AR(2)

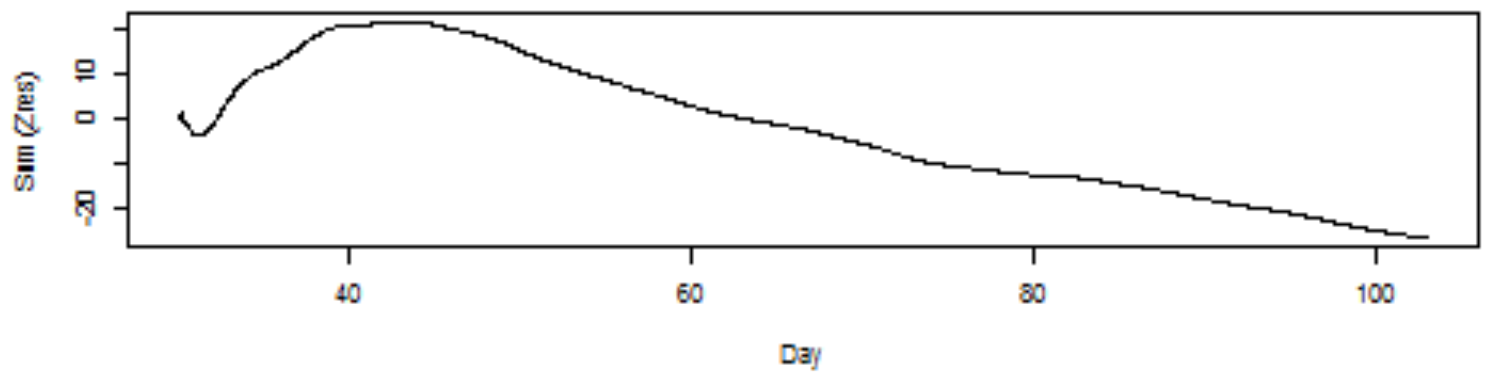

Figure 12. Changes in activity recorded between $8 \mathrm{pm}$ and midnight on 103 successive days for a young male hospitalised for a manic episode on day 104. The top graph shows difference data obtained by a logarithmic transformation of original activity data pooled over five successive observations (10 mins). The middle graph shows changes over time in the logistic transformation of Permutation Entropy estimates computed. The bottom graph shows the accumulated standardized residuals computed using an Adaptive Kalman Filter with an autoregression equation of order 2.

A decrease in $P E$ estimates accompanied by a steady decrease in the accumulated standardised residuals acquired from the Adaptive Kalman Filter analysis indicates the possibility of functional deterioration in this person's mental health from about Day 70 onwards. This change is most evident for activity data recorded in the early morning hours (0000-0400), and to some extent in the 0400-0800 period and in the late evening (20002400). In the afternoon and early evening (1200-1600 and 1600-2000) there is a hint of 
problems around Day 50, but these resolve over time leading to possibly more adaptive behaviour during the afternoon and early evening. A check of medical records would be necessary to determine the clinical significance of these observations. However, at the very least, a combination of an entropy index such as Permutation Entropy and an accompanying change detection tool such as the Adaptive Kalman Filter may have provided early warning to the client and the medical team of the possibility of an imminent serious manic episode.

When $P E$ was used to track complexity of activity over the 103 days, there was a decrease in complexity about two weeks before the young man was hospitalised, as shown in Figure 13. In this case, estimates of $P E$ computed in each moving data window of 30 successive observations $(30 \times 2$ mins $=60$ mins $)$ were standardised to mean zero and standard deviation 1 then added to generate a progression over days. In this analysis, the permutation window, $d$, was five successive observations and the lag, $L$, was 1 . The result shown in Figure 13 suggests that Permutation Entropy could be used to monitor the progress of a chronic disease such as bipolar disorder, and to detect the early signs of relapse that might eventuate in hospitalisation if not addressed as soon as possible after the warning has been given.

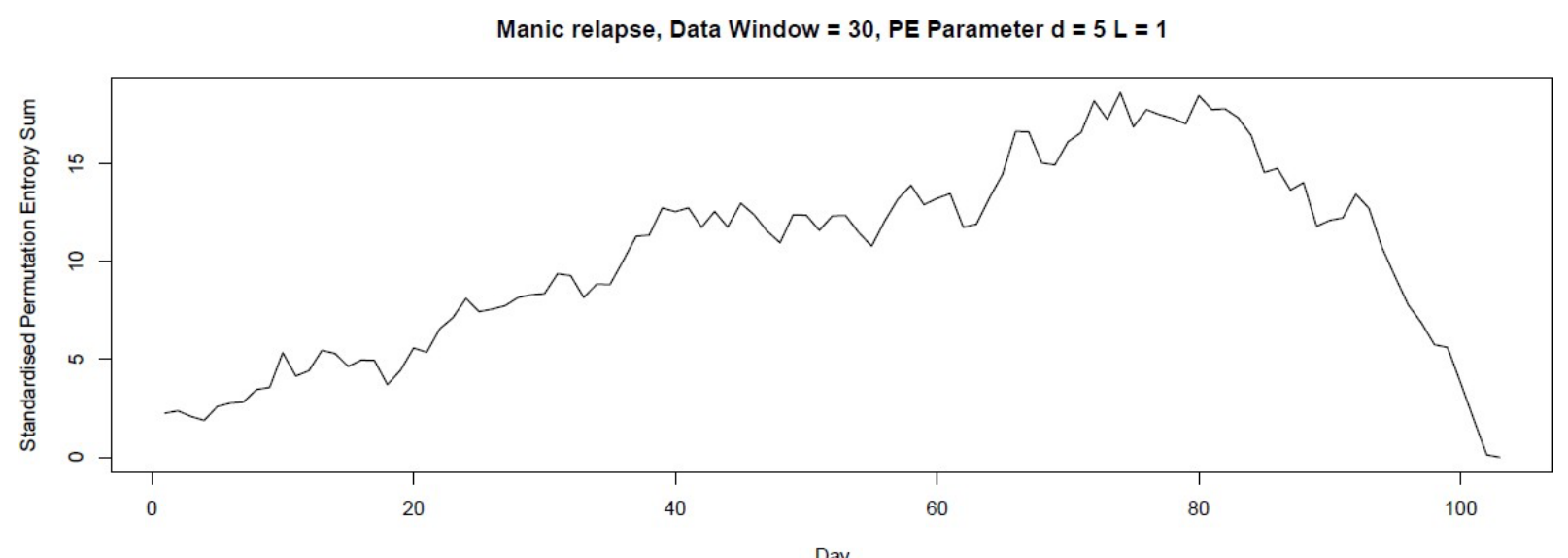

Figure 13. The sum of standardized Permutation Entropy estimates obtained from successive windows of 30 observations (60 mins) with a lag of 1 for 103 days for a young man who was hospitalised with a manic relapse on day 104. 


\section{Conclusions}

The potential for complex nonlinear analyses to advance understanding and management of bipolar disorder is well recognised (for a review, see Bystritsky, Nierenberg, Feusner \& Rabinovich, 2012). Until recently, however, the technology required to non-intrusively collect high-resolution time series data was not widely available or well understood. Such 'big data sets' are now collected routinely worldwide using smartphones, and software engineers are hungry for new clinically-relevant ways to use this large amount of information about human behavior. It is therefore timely for the bipolar disorders field to determine the optimal mathematical characterisation of complexity in risk for bipolar relapse, and we have presented one such approach here. Importantly, by targeting activity rather than self-reported mood, the present approach offers hope of automated monitoring uncontaminated by possible lack of insight and medication adherence issues as a serious episode of bipolar disorder develops.

Following a preliminary analysis using Sample Entropy, the application of Permutation Entropy and an Adaptive Kalman Filter provided additional insight into the progress of a person diagnosed with Bipolar I disorder over and above that available from perusal of the original activity data. Activity data acquired over 103 days provided a rare test bed for examining whether these methodologies could detect precursors of a manic episode that required the patient's hospitalisation on Day 104, at which time the activity record was terminated. In view of circadian effects on mood and activity, the data were analysed separately for each of six four-hour periods. Although intermittent highly variable activity recorded between midnight and 4 am provided a hint of instability between Days 65 and 70, the disturbance was amplified by a decrease in Permutation Entropy and a correspondingly steady decrease in the prediction errors detected by the Adaptive Kalman Filter. Based on this 
further analysis, it would have been reasonable to initiate medical invention by around Day 80 , about three weeks prior to the patient's hospitalisation.

Evidence for activity disturbance was available from the much noisier activity data recorded between 4 am and 8 am, once again suggesting medical intervention around Day 80 . Further evidence of instability occurred during the evening hours from $8 \mathrm{pm}$ to midnight. In this case, the Adaptive Kalman Filter amplified the not so apparent decrease in Permutation Entropy that occurred as early as Day 45. However, this early change in Permutation Entropy was only observed between $8 \mathrm{pm}$ and midnight. It would be interesting to check the clinical record for this patient to determine whether any mood disturbance was noted between Days 45 and 80 , and whether his medical team had provided additional therapeutic interventions during this period. Otherwise, it would be useful to apply these new technologies to help the medical team track the progress of a Bipolar I patient who was susceptible to severe manic episodes.

These results quantify the visual evidence provided in an analysis of this same case by Bullock, Judd and Murray (2014). In their Figure 1, considerable disruption to sleep and activity in the morning hours is apparent from about day 98. Although these authors suggest caution regarding the use of activity data to evaluate sleep in people diagnosed with Bipolar Disorder, a more detailed analysis of the activity data alone can be diagnostically useful provided diurnal changes in activity are considered. The current analyses corroborate the decreased circadian amplitude and dysregulation observed in Figure 15.1 of Murray (2010) using the same clinical data set, while identifying the night-time period as providing potentially more useful prognostic information when compared with daytime activity. As is well-known, a change in activity during the evening and early morning is characteristic of those suffering a manic episode (Grunze, 2010). 
Evidence for a disrupted activity profile about three weeks prior to the manic episode suggests that use of complexity analysis via Permutation Entropy and an associative change detection procedure, such as the Adaptive Kalman Filter, may allow those diagnosed with bipolar disorder and other serious mental illnesses to monitor their progress using one of the commercially available activity measurement devices. An urgent challenge is to develop software for the mobile device so that this methodology can be implemented in real-time.

Although Permutation Entropy and an Adaptive Kalman Filter could be used to predict the onset of a manic episode using activity data obtained from a wearable device, circadian rhythm fluctuations produced differences in predictability during the last few weeks before the manic relapse. As a result, it was difficult to determine precisely when during the day we should record the activity data for the most accurate prediction results. In that analysis we did not employ a statistical decision-making scheme to determine the time at which the relapse process had begun. However, we know that such a scheme is important so that timely medical intervention can occur. This of course is an important issue in the long-term management of people diagnosed with Bipolar I Disorder.

\section{ACKNOWLEDGEMENT}

I thank Professor Greg Murray for kindly providing permission to use his data for this paper. I recognise the significant contribution by all the participants who provided data to help us advance knowledge in the field of mood disorder monitoring.

\section{REFERENCES}

American Psychiatric Association. (2013). Diagnostic and statistical manual of mental disorders: $D S M-5,5$ th ed. Author, Washington, DC. 
Ancoli-Israel, S., Cole, R., Alessi, C., Chambers, M., Moorcroft, W., \& Pollak, C.P. (2003). The role of actigraphy in the study of sleep and circadian rhythms. Sleep, 26, 342392.

Bandt, C., \& Pompe, B. (2002). Permutation entropy: a natural complexity measure for time series. Physics Review Letters, 88, 174102.

Borsboom, D. (2017). A network theory of mental disorders. World Psychiatry, 16, 5-13.

Borsboom, D., Cramer, A.O.J., Schmittmann, V.D., Epskamp, S., \& Waldorp, L.J. (2011). The small world of psychopathology. PLoS One, 17; 6(11):e27407

Boschloo, L., van Borkulo, C.D., Rhemtulla, M., Keyes, K.M., Borsboom, D., \& Schoevers, R.A. (2015). The Network Structure of Symptoms of the Diagnostic and Statistical Manual of Mental Disorders. PLoS ONE 10 (9): e0137621. doi:10.1371/journal.pone.013762

Bullock, B., Judd, F.K., \& Murray, G. (2014). Using actigraphy to monitor sleep-wake patterns in bipolar disorder: a case study. Clinical Psychology Forum, 253, 37-41.

Bullock, B., \& Murray, G. (2014). Reduced amplitude of the 24 hour activity rhythm: A biomarker of vulnerability to bipolar disorder? Clinical Psychological Science, 2, 8696.

Bystritsky, A., Nierenberg, A.A., Feusner, J.D., \& Rabinovich, M. (2012). Computational non-linear dynamical psychiatry: a new methodological paradigm for diagnosis and course of illness. Journal of Psychiatric Research, 46, 428-435.

Cairney, J., Veldhuizen, S., Faulkner, G., Schaffer, A., \& Rodriguez, C. (2009). Bipolar disorder and leisure-time physical activity: results from a national survey of Canadians. Mental Health and Physical Activity, 2, 65-75. 
Cao, Y., Tung, W-w, Gao, J.B., Protopopescu, V.A., \& Hively, L.M. (2004). Detecting dynamical changes in time series using the permutation entropy. Physical Review E, $70,046217$.

Finazzi, M.E., Mesquita, M.E., Lopes, J.R., Fu, L.I., Oliveira, M.G., \& Del Porto, J.A. (2010). Motor activity and depression severity in adolescent outpatients. Neuropsychobiology, 61, 33-40.

Geoffroy, P.A., Etain, B., Sportiche, S., \& Bellivier, F. (2014). Circadian biomarkers in patients with bipolar disorder: promising putative predictors of lithium response. International Journal of Bipolar Disorders, 2, 5. https://doi.org/10.1186/2194-7511-2-5

Geoffroy, P.A., Scott, J., Boudebesse, C., Lajnef, M., Henry, C., Leboyer, M., et al. (2015). Sleep in patients with remitted bipolar disorders: a meta-analysis of actigraphy studies. Acta Psychiatrica Scandinavica, 131, 89-99.

Goodwin, F.K., \& Jamison, K.R. (2007). Manic-depressive illness: Bipolar disorders and recurrent depression. New York: Oxford University Press.

Goodwin, G.M. (2003). Consensus Group of the British Association for Psychopharmacology. Evidence-based guidelines for treating bipolar disorder: recommendations from the British Association for Psychopharmacology. Journal of Psychopharmacology, 17,149-173.

Gottschalk, A., Bauer, M.S., \& Whybrow, P.C. (1995). Evidence of chaotic mood variation in mood disorder. Archives of General Psychiatry, 52, 947-959.

Grunze, H. (2010). The treatment of mania. In Young, A.H., Ferrier, I.N., \& Michelak, E.E. (Eds.) Practical management of bipolar disorder. (pp. 10-23) Cambridge, UK: Cambridge University Press. 
Heath, R.A. (1984). The detection of change in physiological measures using an adaptive Kalman filter algorithm. Psychological Bulletin, 96, 581-588.

Heath, R.A. (2000). Nonlinear dynamics. Techniques and applications in psychology. Mahwah, NJ: Lawrence Erlbaum Associates.

Heath, R.A. (2004). Complexity and mental health. In Holt, T. (Ed.), Complexity for clinicians. (pp. 83-94) Oxford, UK: Radcliffe Medical Press.

Heath, R.A., Heiby, E.M., \& Pagano, I.S. (2007). Complex dynamics in depression: An application to long-term, mood-rating time series. In J. Neufeld (Ed.). Advances in clinical cognitive science: Formal modeling and assessment of processes and symptoms. (pp. 263-292) Washington, D.C.: APA Publications.

Hu, K., Scheer, F.A., Ivanov, P., Buijs, R.M., Shea, S.A. (2007). The suprachiasmatic nucleus functions beyond circadian rhythm generation. Neuroscience, 149, 508-517. Indic, P., Salvatore, P., Maggini, C., Ghidini, S., Ferraro, G., Baldessarini, R.J., Murray, G. (2011). Scaling behavior of human locomotor activity amplitude: Association with bipolar disorder. PLoS ONE, 6(5), e20650. doi:10.1371/journal.pone.0020650

Janney, C. A., Fagiolini, A., Swartz, H. A., Jakicic, J. M., Holleman, R. G., \& Richardson, C. R. (2014). Are adults with bipolar disorder active? Objectively measured physical activity and sedentary behavior using accelerometry. Journal of affective disorders, 152-154, 498-504. https://doi.org/10.1016/j.jad.2013.09.009

Kaplan, K.A., Talbot, L.S., Gruber, J., \& Harvey, A.G. (2012). Evaluating sleep in bipolar disorder: comparison between actigraphy, polysomnography, and sleep diary. Bipolar Disorders, 14, 870-879.

Keller, K., \& Sinn, M. (2005). Ordinal analysis of time series. Physica A, 356, 114-120. 
Krane-Gartiser, K., Henriksen, T.E.G., Morken, G., Vaaler, A., \& Fasmer, O.B. (2014) Actigraphic assessment of motor activity in acutely admitted inpatients with Bipolar Disorder. PLoS ONE, 9(2): e89574. doi:10.1371/journal.pone.0089574

Lam, D., Wong, G. (2005). Prodromes, coping strategies and psychological interventions in bipolar disorders. Clinical Psychology Review, 25, 1028-1042.

Li, X., Ouyang, G., \& Richards, D.A. (2007). Predictability analysis of absence seizures with permutation entropy. Epilepsy Research, 77, 70-74.

Li, J., Yan, J., Liu, X, \& Ouyang, G. (2014). Using permutation entropy to measure the changes in EEG signals during absence seizures. Entropy, 16, 3049-3061.

Merikangas, K.R., Jin, R., He, J.P., Kessler, R.C., Lee, S., Sampson, N.A., et al. (2011). Prevalence and correlates of bipolar spectrum disorder in the world mental health survey initiative. Archives of General Psychiatry, 68, 241-251.

Minassian, A., Henry, B.L., Young, J.W., Masten, V., Geyer, M.A., \& Perry, W. (2011). Repeated assessment of exploration and novelty seeking in the Human Behavioral Pattern Monitor in Bipolar Disorder patients and healthy individuals. PLoS ONE, 6(8): e24185. https://doi.org/10.1371/journal.pone.0024185

Moore, P.J., Little, M.A., McSharry, P.E., Geddes, J.R., \& Goodwin, G.M. (2012). Forecasting depression in bipolar disorder. IEEE Transactions on Biomedical Engineering, 59, 2801-2807.

Moore, P.J., Little, M.A., McSharry, P.E., Goodwin, G.M., \& Geddes, J.R. (2014). Mood dynamics in mood disorder. International Journal of Bipolar Disorders, doi:10.1186/s40345-014-0011-z.

Murray, G. (2007). Diurnal mood variation in depression: a signal of disturbed circadian function? Journal of Affective Disorders, 102, 47-53. 
Murray, G. (2010). Circadian and sleep/wake considerations in the practical management of bipolar disorder. In Young, A.H., Ferrier, I.N., \& Michelak, E.E. (Eds.) Practical management of bipolar disorder. (pp. 152-162) Cambridge, UK: Cambridge University Press.

Murray, G., Allen, N.B., Trinder, J., Burgess, H. (2002). Is weakened circadian rhythmicity a characteristic of neuroticism? Journal of Affective Disorders, 72, 281-289.

Olofsen, E., Sleigh, J.W., \& Dahan, A. (2008). Permutation entropy of the electroencephalogram: a measure of anaesthetic drug effect. British Journal of Anaesthesia, 101, 810-821.

R Core Team (2021). R: A language and environment for statistical computing. R Foundation for Statistical Computing, Vienna, Austria. URL https://www.R-project.org/

Ratheesh, A., Berk, M., Davey, C.G., McGorry, P.D., \& Cotton, S.M. (2015). Instruments that prospectively predict bipolar disorder - A systematic review. Journal of Affective Disorders, 179, 65-73.

Richman, J.S., \& Moorman, J.R. (2000). Physiological time series analysis using approximate entropy and sample entropy. American Journal of Physiology: Heart Circulation Physiology, 278, H2039-H2049.

Santaniello, S., Burns, S.P., Golby, A.J., Singer, J.M., Anderson, W.S., \& Sarma, S.V. (2011). Quickest detection of drug-resistant seizures: An optimal control approach. Epilepsy \& Behavior, 22, S49-S60.

Tahmasian, M., Khazaie, H., Golshani, S., \& Avis, K.T. (2013). Clinical application of actimetry in psychotic disorders: A systematic review. Current Psychiatry Reports, $15,359-373$. 


\section{APPENDIX}

Routines to estimate Sample Entropy, Permutation Entropy and the Adaptive Kalman Filter were programed in the R Statistical Language (R Core Team, 2021) by RH.

\section{Sample Entropy}

Sample Entropy indicates how similar a time series is over a sequence of observations. The more similar the observations are, the less complex is the process that generates the time series. In this way Sample Entropy serves as a useful complexity index. Sample Entropy is defined as the negative natural logarithm of the conditional probability that two sequences that are similar after $m$ observations remain similar at the next time point (Richman \& Moorman, 2000).

Consider a sequence of $N$ relatively uncorrelated data values, $x_{1}, x_{2}, x_{3}, \ldots, x_{N}$. Embed this series in an m-dimensional space by creating vectors such as

$$
X_{m}(i)=\left\{x_{i}, x_{i+1}, x_{i+2}, \ldots, x_{i+m-1}\right\}
$$

and compute any distance function (a city-block metric will do) between any two of these embedded vectors, $d\left[X_{m}(i), X_{m}(j)\right], i \neq j$. Then Sample Entropy $=-\log \frac{A}{B}$, where $A$ is the number of embedded vector pairs for which $\left[X_{m+1}(i), X_{m+1}(j)\right]<r$, and $B$ is the number of embedded vector pairs for which $\left[X_{m}(i), X_{m}(j)\right]<r$. When the time series has been standardised with mean 0 and standard deviation $1, m=2$ and $r=0.2$.

\section{Permutation Entropy}

The data to be analysed are represented by a sequence of $N$ relatively uncorrelated data values, $x(1), x(2), \ldots, x(\mathrm{t}), \ldots, x(N)$. The moving data window of width $w$ initially contains the 
first subset of observations $x(1), x(2), \ldots, x(w)$. The next data window moves along one time unit to produce $x(2), x(3), \ldots, x(w), x(w+1)$. For a data set containing $N$ observations there are $\left(N^{*}-1\right)$ data windows, where $N^{*}$ is the largest multiple of $w$ that does not exceed $N$. The Permutation Entropy (PE) for window $w, P E[w]$, is computed using Eq. (A1), where $\pi(i)$, $i=1, d$ !, represents the $i$-th of $d$ ! possible permutations of the data within the window ending with observation $x(w) . d$ ! is obtained by multiplying successive integers from 1 to $d$.

$$
P E[w, i]=-\frac{\sum_{i=1}^{d !} p(w, i) \log (p(w, i))}{\log (d !)}, p(w, i) \neq 0
$$

where $p(w, i)$ is the proportion of permutations, $\pi(i)$, in a data window of width $w$. The sum in Eq. (A1) is computed over all possible permutations of numbers within a window of permutation length, $d$. Changes in $P E$ over time are shown by plotting $P E[w, i]$ as the window, $w$, moves forward in time.

$P E$ remains unchanged when the data are transformed by an order-preserving relationship, such as a logarithmic transformation. $P E$ is sensitive to temporal changes in the statistical properties of a time series and is relatively simple to compute. $P E$, as defined in Eq. (A1), ranges from 0 to 1 . When $P E$ is close to the extreme values of 0 and 1 , a more suitable index is obtained by computing the logistic transformation, $\operatorname{logit}(P E)=\log [(P E) /(1-P E)]$. This transformed value, which now ranges from $-\infty$ to $+\infty$, is used in the $P E$ analyses described in this paper.

\section{A Simple Example of How Permutation Entropy is Computed}

This example examines changes over time in activity using permutation length, $d=3$, and lag, $L=1$. The data and preliminary analysis are shown in the following table. There are $N=12$ observations and the window length, $w$, is equal to 3 . The data window at each successive time is indicated by the bold-faced numbers in each row. At the end of each row, the order of these bold observations in the data window is shown (lowest to highest values). 
$\begin{array}{lc}\mathbf{8 1}, \mathbf{8 3}, \mathbf{7 6}, 77,63,64,74,80,84,83,77,75 & \text { order }=(2,3,1) \\ 81, \mathbf{8 3}, \mathbf{7 6}, \mathbf{7 7}, 63,64,74,80,84,83,77,75 & \text { order }=(3,1,2) \\ 81,83, \mathbf{7 6}, \mathbf{7 7}, \mathbf{6 3}, 64,74,80,84,83,77,75 & \text { order }=(2,3,1) \\ 81,83,76, \mathbf{7 7 , 6 3}, \mathbf{6 4}, 74,80,84,83,77,75 & \text { order }=(3,1,2) \\ 81,83,76,77, \mathbf{6 3}, \mathbf{6 4}, \mathbf{7 4}, 80,84,83,77,75 & \text { order }=(1,2,3) \\ 81,83,76,77,63, \mathbf{6 4}, \mathbf{7 4}, \mathbf{8 0}, 84,83,77,75 & \text { order }=(1,2,3) \\ 81,83,76,77,63,64, \mathbf{7 4}, \mathbf{8 0}, \mathbf{8 4}, 83,77,75 & \text { order }=(1,2,3) \\ 81,83,76,77,63,64,74, \mathbf{8 0}, \mathbf{8 4}, \mathbf{8 3}, 77,75 & \text { order }=(1,3,2) \\ 81,83,76,77,63,64,74,80, \mathbf{8 4}, \mathbf{8 3}, 77,75 & \text { order }=(3,2,1) \\ 81,83,76,77,63,64,74,80,84, \mathbf{8 3}, \mathbf{7 7}, 75 & \text { order }=(3,2,1)\end{array}$

The relative frequency of each order and its contribution to the sum in Eq. (A1), $-p \log (p)$, is as follows.

Order Frequency Proportion $(p) \quad-p \log (p)$

$\begin{array}{llll}(1,2,3) & 3 & 0.3 & 0.156 \\ (1,3,2) & 1 & 0.1 & 0.100 \\ (2,1,3) & 0 & & \\ (2,3,1) & 2 & 0.2 & 0.140 \\ (3,1,2) & 2 & 0.2 & 0.140 \\ (3,2,1) & 2 & 0.2 & 0.140\end{array}$

Using Eq. (A1), Permutation Entropy is computed using:

$\operatorname{Sum}(-p \log (p))=0.676$

Permutation Entropy $=\operatorname{Sum}(-p \log (p)) / \log (3 !)=0.676 / 0.778=0.869$

\section{An Autoregressive Model}

An autoregressive model of order $r$ has the $(m+1)$-th observation from a series $x_{1}, x_{2}, x_{3}, \ldots$, $x_{N}$ being predicted from a linear combination of the previous $r$ observations. 


$$
x_{m+1}=\sum_{i=1}^{r} a_{i} x_{m-i}+a_{0}
$$

where the $a_{i}, i=0, r$ are chosen so that the mean square prediction error of $x_{m+1}$ is minimized.

\section{An Adaptive Kalman Filter}

The Adaptive Kalman Filter operates in a similar manner to a thermostat. Once the operating temperature, or set point, has been set, the thermostat applies heat when the ambient temperature is below the set point and cooling when the ambient temperature is above the set point. The direction and size of the correction is proportional to the difference between the ambient temperature and the set point in both cases. The Adaptive Kalman Filter extends this idea by allowing the coefficients of a prediction equation, the autoregression parameters, to be adjusted so that the difference between the set point and any new observation, the residual or prediction error, is minimised. Any change in the temporal characteristics of a sequence of observations will require adjustment of autoregression parameters to bring under control a temporary increase in the volatility of the prediction errors.

This example follows that contained in Heath (2000, pp. 33-34). Ten observations were computed using the autoregression equation: $A(i+2)=0.5 A(i+1)-0.5 A(i), i=1,8$. This equation uses two autoregression parameters, equal to 0.5 and -0.5 , respectively. An autoregression equation is like an ordinary linear regression equation except that the predictors are previous observations in the time series. After adding normally distributed measurement errors with mean zero and standard deviation 0.1 , the following observation series was obtained by iterating the autoregression equation for $i$ ranging from 1 to 8:

$$
A=\{0.119,0.001,0.128,-0.098,-0.064,-0.030,-0.231,-0.145,-0.071,-0.135\} \text {. }
$$

The first guess for the autoregression parameters is given by dividing 1 by the number of parameters so that their sum equals 1 . In this example the starting parameter list is 
$a=\{0.5,0.5)$. An estimate of the third observation based on current knowledge of the parameters is given by $\operatorname{Est}[A(3)]=0.5 \times 0.001+0.5 \times 0.119=0.060$.

The prediction error, $e(3)$, is obtained by subtracting Est $[A(3)]$ from $A(3)$ to produce

$$
e(3)=0.128-0.060=0.068 \text {. }
$$

The matrix operations described in Heath (1984) result in an update of the autoregression parameters to produce $a(1)=0.500, a(2)=0.508$, this adjustment being in proportion to the size of the estimation error and the gain multiplier, an estimate of how much change is needed to track future observations more closely. This process continues until the estimates of the autoregression parameters approach their true values of $a(1)=0.500, a(2)=-0.500$. If there is a change in these parameters, the prediction errors become large, and it takes some time for the system to converge towards the new autoregression parameter values. In the software used for analyses, a useful index of change in the underlying system parameters is the accumulated standardized prediction errors or residuals, each having mean 0 and standard deviation 1. 